\title{
Nonlocal free energy of a spatially inhomogeneous superconductor.
}

\author{
K.V. Grigorishin* and B.I. LeN \\ Boholyubov Institute for Theoretical Physics of the Ukrainian National \\ Academy of Sciences, 14-b Metrolohichna str. Kiev-03680, Ukraine.
}

(Dated: October 31, 2018)

\begin{abstract}
The microscopic approach was developed for obtaining of the free energy of a superconductor based on direct calculation of the vacuum amplitude. The free energy functional of the spatially inhomogeneous superconductor in a magnetic field was obtained with help of the developed approach. The obtained functional is generalization of Ginzburg-Landau functionals for any temperature, for arbitrary spatial variations of the order parameter and for the nonlocality of a magnetic response and the order parameter. Moreover, the nonlocality of the magnetic response is the consequence of order parameter's nonlocality. The extremals of this functional are considered in the explicit form in the low- and high-temperature limit at the condition of slowness of spatial variations of the order parameter.

Keywords: uncoupling of correlations, vacuum amplitude, order parameter, magnetic response, nonlocality.

PACS numbers: 74.20.-z, 74.20.De, 74.20.Fg, 64.60.Bd
\end{abstract}

\section{INTRODUCTION}

One of the important applications of condensed matter physics is the description of thermodynamics and electrodynamics of superconductors. Several types of superconducting materials are spatially inhomogeneous anisotropic structures. In particular, heterostructures and superlattice, combinations of superconductors with the materials with different types of a conductivity. The superconductive and transport properties of the inhomogeneous systems is determined by the proximity effect, which results to modulation of the order parameter, and by Josephson effect [1, 2]. At the present moment many various heterostructures have been made on the basic of the high temperature superconductors (HTS). However the HTS can be considered as the heterostructures 3], where the conducting $\mathrm{CuO}_{2}$ layers play a role quantum wells, and the layers of atoms between them (the reservoirs of charge carrier) play a role quantum barriers. The value of critical temperature can essentially depend on the spatial structure of the superconducting system. So, in the papers [6-8] the model of cluster crystal (the lattice is formed by metallic clusters) with the giant intensification of a superconductive pairing (hypothetically the critical temperature is $T_{C} \geq 150 K$ ) was proposed. Such crystal can be considered as a superconducting superlattice. The intensification of Cooper pairing can occur in a neighborhood of the special defects in a solid [9].

The modification of Ginzburg-Landau equation is necessary for description of spatially inhomogeneous superconducting systems. So, the modified functional of free anergy is proposed for the description of influence of the defects [9], where the term $-\gamma \delta(x)|\Delta(x)|^{2}$ is introduced ( $\gamma$ is coupling constant, $\delta(x)$ - Dirac function, $|\Delta(x)|$ is the gap function - order parameter). This term considers the intensification of Cooper pairing around the defect, moreover the area of intensification is much less than the correlation length $l_{0}$ of the superconductor. For description of layered compounds of type HTS and $M g B_{2}[4]$ the modified Eliashberg equations with consideration of anisotropy and Lawrence-Doniach functional [5] for quasi two dimensional superconductors are applied. The nonlocality causes additional complication in the description of the spatially inhomogeneous systems. The superconducting systems with a deep modulation of the order parameter must be considered as the systems of Josephson contacts. For the structures where $d \gg \Lambda$ ( $d$ is the thickness of a contact, $\Lambda$ is the magnetic penetration depth) the nonlocality is the determining factor of electrodynamics effects [10 12]. However the effects of nonlocality can be essential even for the cases when $d \ll \Lambda$ [13 15].

For the complete description of a superconductor it is necessary to know the functional of free energy $\Omega(\beta, \Delta, \mathbf{A})$, where $\beta=\frac{1}{T}$ is the inverse temperature, $\Delta$ is the gap function (order parameter), $\mathbf{A}$ is the potential of microscopic magnetic field. Then the equations $\frac{\delta \Omega}{\delta \Delta}=0, \frac{\delta \Omega}{\delta \mathbf{A}}=0$ are the equations of state of a superconductor. The basic method for obtaining of the equations of state is solution of Gor'kov equations or Bogoliubov equations with the equation

*Electronic address: konst’dark@mail.ru

${ }^{\dagger}$ Electronic address: bohdan.lev@gmail.com 
of self-consistency for the order parameter [16] $\Delta(\mathbf{r})$ and $\Delta^{+}(\mathbf{r})$. However this set of equations is nonlinear. If the temperature is close to the critical temperature $T \rightarrow T_{C} \Rightarrow \Delta \rightarrow 0$, then these equations can be represented in the form of series in degrees of $\Delta$. Moreover, magnetic penetration depth $\Lambda$ must be larger then Pippard coherent length $l_{0}$, hence the potential A changes a little on the coherent length. As a result we have the well-known Ginzburg-Landau equations. The equations are correct for description of thermodynamics and electrodynamics of a superconductor under the following restrictions:

1. The energy gap is much less than the critical temperature. Then the parameter $\Delta(\mathbf{r}, T) / T_{C} \ll 1$ can be expansion parameter. This means, that the equations are correct in the range $T \rightarrow T_{C}$ or in the range $H \rightarrow H_{C 2}$ (intensity of magnetic field is close to the second critical magnetic field $H_{C 2}$ ).

2. $\Delta(\mathbf{r}, T)$ changes slowly on the coherent length $l(T)$, which is size of a Cooper pair.

3. Magnetic field $\mathbf{H}(\mathbf{r})=\operatorname{rot} \mathbf{A}(r)$ changes slowly on the coherent length, that is the magnetic penetration depth is $\Lambda(T) \gg l(0)$. This means, that electrodynamics of a superconductor is local.

In the papers [17, 18] the equations have been proposed, where the first restriction is absent. These equations were obtained from Gor'kov equations and they are the generalization of Ginzburg-Landau equations for the case of arbitrary value of $\Delta(\mathbf{r}, T) / T$. However spatial inhomogeneities are slow, the order parameter and magnetic response are local. In the Ref. [19] on basic Bogoliubov equation it was shown that if the gap $\Delta(\mathbf{r})$ varies with position, that the value of the gap in a point is determined by a distribution of gap in some neighborhood: $\Delta(\mathbf{r})=\int d \mathbf{r}^{\prime} Q\left(\mathbf{r}, \mathbf{r}^{\prime}\right) \Delta^{\prime}(\mathbf{r})$, moreover the kernel $Q$ can be function of the order parameter $\Delta\left(\mathbf{r}^{\prime}\right)$ also. This means that the order parameter is nonlocal.

The problem of description of the superconducting phase can be solved by another method. This method is the direct calculation of free energy. To this end we must calculate the partition function $Z$

$$
Z=S p\left(Z_{0} \widehat{\rho}_{0} \widetilde{U}(\beta)\right)=Z_{0} R(\beta)
$$

where $Z_{0}$ is the partition function for a system of noninteracting particles, $\widetilde{U}(\beta)=e^{+\beta\left(\widehat{H}_{0}-\mu \widehat{N}\right)} e^{-\beta(\widehat{H}-\mu \widehat{N})}$ is the evolution operator in the interaction representation (it describes evolution of the system in imaginary time $i t \rightarrow \tau$, $\tau \in[0, \beta]), \widehat{H}=\widehat{H}_{0}+\widehat{H}_{I}$ is the system Hamiltonian, $\widehat{N}$ is the particle operator, $\mu$ is the chemical potential (the replacement of Hamiltonian $\widehat{H}$ in canonical ensemble by Hamiltonian $\widehat{H}-\mu \widehat{N}$ in grand canonical ensemble leads to the shift of reference of particle's energy from zero to Fermi surface: $\left.\varepsilon\left(k_{F}\right)=0\right)$,

$$
R(\beta)=\langle\widehat{U}(\beta)\rangle_{0}=S p\left(\widehat{\rho}_{0} \widetilde{U}(\beta)\right)=\sum_{n=0}^{\infty} \frac{(-1)^{n}}{n !} \int_{0}^{\beta} d \tau_{1} \ldots \int_{0}^{\beta} d \tau_{n} S p\left(\widehat{\rho}_{0} \widehat{T}\left\{\widehat{H}_{1}\left(\tau_{I}\right) \ldots \widehat{H}_{I}\left(\tau_{n}\right)\right\}\right)
$$

is the vacuum amplitude of the system, where $\widehat{H}_{I}(\beta)=e^{+\beta\left(\hat{H}_{0}-\mu \hat{N}\right)} \widehat{H}_{I} e^{-\beta\left(\hat{H}_{0}-\mu \hat{N}\right)}$ is the interaction operator of particles in interaction representation, $\widehat{T}$ is the ordering operator in time. The averaging \langle\rangle$_{0} \equiv S p\left(\widehat{\rho}_{0} \ldots\right)$ is done over ensemble of noninteracting particles.

The partition function $Z_{0}$ can be found exactly for any system. If particles interact, then the situation becomes essentially complicated. Solution of the problem reduces to the calculation of a transition amplitude "vacuum-vacuum" (2). The vacuum amplitude determines the internal energy of a system:

$$
U=-\frac{\partial}{\partial \beta} \ln Z_{0}-\frac{\partial}{\partial \beta} \ln R(\beta)
$$

and the grand thermodynamical potential:

$$
\Omega=-\frac{1}{\beta} \ln Z_{0}-\frac{1}{\beta} \ln R(\beta)
$$

The potential $\Omega$ plays a role of Helmholtz free energy if a particle's energy is counted from Fermi surface. Therefore we will call the grand thermodynamical potential as free energy for brevity.

Eqs. (3/4) are valid if the symmetries of ground state of the system with interaction $\left|\Psi_{0}\right\rangle$ and without interaction $\left|\Phi_{0}\right\rangle$ are identical: $\left\langle\Phi_{0} \mid \Psi_{0}\right\rangle \neq 0$. This means that: 1)the interaction potential is being switched slowly in the system in the ground state without interaction, 2) the ground state of system with interaction is being obtained by continuous way from the ground state without interaction while the switching of the interaction (adiabatic hypothesis) 20, 21]. If the wave functions are orthogonal $\left\langle\Phi_{0} \mid \Psi_{0}\right\rangle=0$ - the adiabatic hypothesis is not valid, and the symmetries of the 
system with interaction and without one are different. This means, that the initial system without interaction suffers a phase transition stipulated by the interaction. For the system with the broken symmetry we can calculate the vacuum amplitude on the free propagators $G_{0}(\mathbf{k}, \tau)$ and use Eqs. (3/4). However we will find the wrong thermodynamics potentials. This means that the state exists with more low energy than the obtained value.

The method for direct calculation of vacuum amplitude $R(\beta)$ has been proposed in Ref. 222]. The concept lies in the fact that we consider electrons in a normal metal propagating in a random "field" of fluctuations of the order parameter $\Delta_{\mathbf{q}}$, where $\mathbf{q}$ is small wave-vector. The operator of the interaction of electrons with the fluctuations can be written as:

$$
\widehat{H}_{i n t}=\sum_{\mathbf{p}}\left[\Delta_{\mathbf{q}} \widehat{C}_{\mathbf{p}_{+}}^{+} \widehat{C}_{-}^{+} \mathbf{p}_{-}+\Delta_{\mathbf{q}}^{*} \widehat{C}_{-\mathbf{p}_{-}} \widehat{C}_{\mathbf{p}_{+}}\right]
$$

where $\mathbf{p}_{ \pm}=\mathbf{p} \pm \mathbf{q} / 2$. The correction to the thermodynamics potential is represented via the vacuum amplitude $R$ utilizing Wick theorem:

$$
\begin{aligned}
\Delta \Omega & =-T \ln R(\beta) \approx-T[R(\beta)-1]=-\frac{T}{2 !} \int_{0}^{1 / T} d \tau_{1} \int_{0}^{1 / T} d \tau_{2}\left\langle\widehat{T}_{\tau}\left(\widehat{H}_{i n t}\left(\tau_{1}\right) \widehat{H}_{i n t}\left(\tau_{2}\right)\right)\right\rangle+\ldots \\
& =-T \int_{0}^{1 / T} d \tau_{1} \int_{0}^{1 / T} d \tau_{2}\left|\Delta_{\mathbf{q}}\right|^{2} \sum_{\mathbf{p}} G_{0}\left(\mathbf{p}_{+}, \tau_{1}-\tau_{2}\right) G_{0}\left(-\mathbf{p}_{-}, \tau_{1}-\tau_{2}\right)+\ldots
\end{aligned}
$$

The correction $\Delta \Omega$ is represented via the free propagators $G_{0}$ of normal state only - we consider normal metal at $T>T_{C}$, where the fluctuation sourse of Cooper pair (36) acts. As a result we have Landau expansion:

$$
\Omega_{s}-\Omega_{n}=\sum_{\mathbf{q}}\left[\alpha(T)\left|\Delta_{\mathbf{q}}\right|^{2}+\frac{b}{2}\left|\Delta_{\mathbf{q}}\right|^{4}+\gamma q^{2}\left|\Delta_{\mathbf{q}}\right|^{2}\right],
$$

where $\alpha(T) \propto\left(T-T_{C}\right), b, \gamma$ are the expansion coefficients. As for calculation of the correction $\Delta \Omega$ the free propagators $G_{0}$ of normal phase is used only, the condensed phase is considered as fluctuations against the background of the normal phase. This means, that the high-temperature limit of superconductor's free energy functional $\Omega\left(T \rightarrow T_{C}\right)$ can be obtained by this method only. Moreover, the hamiltonian (5) does not contain any parameters of a matter (for example, interaction constant between fermions). In the present paper we proposed the method, which permits to calculate the free energy functional of a spatial inhomogeneous superconductor for any temperature. The vacuum amplitude $R(\beta)$ is calculated on the interaction between particles, that allows to use model potentials. This gives a possibility to build the free energy functional of spatial inhomogeneous superconductor in external fields proceeding from first principles, unlike the method presented in [22] where $R(\beta)$ is calculated on interaction of particles with a random "field" of fluctuations of the order parameter $\Delta_{\mathbf{q}}$, and the critical temperature $T_{C}$ is introduced by phenomenologically (its connection with the interaction between particle is absent). Our method can be generalized to second order phase transition in other systems, because any phase transitions can be described in the formalism of the anomalous Green function [21].

In the presented paper we have developed the microscopic approach for finding of free energy functional of a superconductor $\Omega(\Delta, T)$ with help of direct calculation of the vacuum amplitude. This value is calculated on the dressed one-particle propagators. The propagators are dressed due to the interaction of free fermions with the fluctuations of pairing by the method presented in [23, 24]. In the sections III] and IV] using the developed approach of microscopic description of superconducting phase we obtain the functional of free energy of a spatial inhomogeneous superconductor in a magnetic field $\Omega\left(\Delta_{\mathbf{q}}, \mathbf{a}(\mathbf{q}), T\right)$. This functional generalizes Ginzburg-Landau functional for arbitrary temperatures, for arbitrary spatial variations of the order parameter and for nonlocality of the order parameter and a magnetic response.

\section{THE UNCOUPLING OF CORRELATIONS METHOD FOR CALCULATION OF A VACUUM AMPLITUDE.}

\section{A. Normal and anomalous propagators.}

Let we have the system of $N$ noninteracting fermions in volume $V$ at temperature $T$. For the description of this system we use Matsubara propagators, where time $t$ is complex: $t \rightarrow-i \tau, \tau \in[0, \beta]$. In ideal Fermy gas propagation 
of a particle with momentum $\mathbf{k}$, energy $\varepsilon \approx v_{F}\left(|\mathbf{k}|-k_{F}\right)$ counted from Fermy surface (we are using system of units, where $\hbar=k_{B}=1$ ) and with spin $\sigma$ is described by the free propagator:

$$
\begin{aligned}
& G_{0}\left(\mathbf{k}, \tau=\tau_{2}-\tau_{1}\right)=\left\{\begin{array}{cc}
-i \operatorname{Sp}\left\{\widehat{\rho}_{0} C_{\mathbf{k}, \sigma}\left(\tau_{2}\right) C_{\mathbf{k}, \sigma}^{+}\left(\tau_{1}\right)\right\}, & \tau>0 \\
i \operatorname{Sp}\left\{\widehat{\rho}_{0} C_{\mathbf{k}, \sigma}^{+}\left(\tau_{1}\right) C_{\mathbf{k}, \sigma}\left(\tau_{2}\right)\right\}, & \tau \leq 0
\end{array}\right\}= \\
& -i \theta_{\tau}\left(g_{0}^{+} A_{0} e^{-|\varepsilon| \tau}+g_{0}^{-} B_{0} e^{|\varepsilon| \tau}\right)+i \theta_{-\tau}\left(g_{0}^{-} A_{0} e^{-|\varepsilon| \tau}+g_{0}^{+} B_{0} e^{|\varepsilon| \tau}\right), \quad \theta_{\tau}=\left\{\begin{array}{ll}
1, & \tau>0 \\
0, & \tau<0
\end{array}\right\}, \\
& G(\mathbf{k}, \tau)=\frac{1}{\beta} \sum_{n=-\infty}^{n=+\infty} G\left(\mathbf{k}, \omega_{n}\right) e^{-i \omega_{n} \tau}, \quad G\left(\mathbf{k}, \omega_{n}\right)=\frac{1}{2} \int_{-\beta}^{\beta} G(\mathbf{k}, \tau) e^{i \omega_{n} \tau} d \tau, \quad \omega_{n}=\frac{(2 n+1) \pi}{\beta}, \\
& G_{0}\left(\mathbf{k}, \omega_{n}\right)=\frac{i}{i \omega_{n}-\varepsilon(k)}=i \frac{i \omega_{n}+\varepsilon}{\left(i \omega_{n}\right)^{2}-\varepsilon^{2}}=i \frac{A_{0}}{i \omega_{n}-|\varepsilon|}+i \frac{B_{0}}{i \omega_{n}+|\varepsilon|},
\end{aligned}
$$

where

$$
A_{0}=\frac{1}{2}\left(1+\frac{\varepsilon}{|\varepsilon|}\right), \quad B_{0}=\frac{1}{2}\left(1-\frac{\varepsilon}{|\varepsilon|}\right), \quad g_{0}^{+}=\frac{1}{e^{-|\varepsilon| \beta}+1}, \quad g_{0}^{-}=\frac{1}{e^{|\varepsilon| \beta}+1},
$$

$C_{\mathbf{k}, \sigma}(\tau)$ and $C_{\mathbf{k}, \sigma}^{+}(\tau)$ are creation and annihilation operators in Heisenberg representation, $\widehat{\rho}_{0}$ is the density matrix of noninteracting particles:

$$
\widehat{\rho}_{0}=\exp \left\{\frac{\Omega-\widehat{H}_{0}}{T}\right\}=\exp \left\{\frac{\Omega-\sum_{\mathbf{k}, \sigma} \varepsilon(k) C_{\mathbf{k}, \sigma}^{+} C_{\mathbf{k}, \sigma}}{T}\right\} .
$$

Now let an attracting force acts between particles. Hamiltonian of the system is

$$
\widehat{H}_{0}+\widehat{H}_{I}=\sum_{\alpha} \sum_{\mathbf{k}} \varepsilon(\mathbf{k}) C_{\mathbf{k}, \alpha}^{+} C_{\mathbf{k}, \alpha}+\frac{1}{2 V} \sum_{\alpha, \gamma} \sum_{\mathbf{k}, \mathbf{l}} V_{\mathbf{l},-\mathbf{l}, \mathbf{k},-\mathbf{k}} g_{\alpha \gamma} C_{-\mathbf{l}, \gamma}^{+} C_{\mathbf{l}, \alpha}^{+} C_{\mathbf{k}, \alpha} C_{-\mathbf{k}, \gamma}
$$

The force is described by matrix element of interaction potential:

$$
\langle\mathbf{l},-\mathbf{l}|\widehat{V}| \mathbf{k},-\mathbf{k}\rangle=\lambda w_{l} w_{k}<0, \quad w_{k}=\left\{\begin{array}{ll}
1, & \varepsilon(k)<\omega_{D} \\
0, & \varepsilon(k)>\omega_{D}
\end{array}\right\},
$$

moreover interacting particles have opposite spins: $g_{\alpha \gamma}=\left\{\begin{array}{ll}1, & \alpha \neq \gamma \\ 0, & \alpha=\gamma\end{array}\right\}$.

The interaction (12) leads to Cooper instability. Let the additional particle with momentum $\{\mathbf{k}, \omega\}$ propagates through the system of identical fermions. A some pair of fermions decays in components with momenta $\{-\mathbf{k},-\omega\}$ and $\{\mathbf{k}, \omega\}$ with amplitude $i \Delta^{+}$. The second particle of the decayed pair is in a state of the additional particle (k, $\left.\omega\right)$ and it is identical to the additional particle. The second particle propagates through the system further. The first particle of decayed pair forms bound state with the initial additional particle with amplitude $-i \Delta$. Anew formed pair replenishes the condensate of pairs in the system. Thus, the dressed propagator $G_{S}$ considers the interaction of a particle with the fluctuations of pairing. Intensity of the interaction is given by the amplitudes $-i \Delta$ and $i \Delta^{+}$. Therefore we can write the mass operator for such process (Fig 10 as [23, 24]

$$
-\Sigma\left(\mathbf{k}, \omega_{n}\right)=(-\Delta) i G_{0}^{+}\left(-\mathbf{k}, \omega_{n}\right)\left(-\Delta^{+}\right)=\frac{-\Delta^{+}}{i \omega_{n}+\varepsilon(k)}
$$

The dressed propagator can be found from Dyson equation:

$$
\begin{aligned}
& \frac{1}{G_{0}}=\frac{1}{G_{S}}-i \Sigma \Rightarrow G_{S}\left(\mathbf{k}, \omega_{n}\right)=\frac{i}{i \omega_{n}-\varepsilon(k)-\Sigma\left(\mathbf{k}, \omega_{n}\right)} \\
& =i \frac{i \omega_{n}+\varepsilon}{\left(i \omega_{n}\right)^{2}-E^{2}(k)}=i \frac{A_{S}}{i \omega_{n}-E(k)}+i \frac{B_{S}}{i \omega_{n}+E(k)},
\end{aligned}
$$




\section{$-\Sigma=-\triangle \overbrace{}^{i \sigma_{0}^{+}\left(-k_{,} \omega_{n}\right)}(-4-$}

Figure 1: The diagram for the mass operator $\Sigma$ describing interaction of a fermion with fluctuations of pairing.

where $E=\sqrt{\varepsilon^{2}(k)+\Delta \Delta^{+}}$. It can be written with help of a total definition of Green function in $(\mathbf{k}, t)$-space:

$$
\begin{aligned}
& \left.G_{S}(\mathbf{k}, \tau)=\tau_{2}-\tau_{1}\right)=\left\{\begin{array}{cc}
-i \operatorname{Sp}\left\{\widehat{\varrho}_{0} C_{\mathbf{k}, \sigma}\left(\tau_{2}\right) C_{\mathbf{k}, \sigma}^{+}\left(\tau_{1}\right)\right\}, & \tau>0 \\
i \operatorname{Sp}\left\{\widehat{\varrho}_{0} C_{\mathbf{k}, \sigma}^{+}\left(\tau_{1}\right) C_{\mathbf{k}, \sigma}\left(\tau_{2}\right)\right\}, & \tau \leq 0
\end{array}\right\} \\
& =-i \theta_{\tau}\left(g_{S}^{+} A_{S} e^{-E \tau}+g_{S}^{-} B_{S} e^{E \tau}\right)+i \theta_{-\tau}\left(g_{S}^{-} A_{S} e^{-E \tau}+g_{S}^{+} B_{S} e^{E \tau}\right),
\end{aligned}
$$

where

$$
A_{S}=\frac{1}{2}\left(1+\frac{\varepsilon}{E}\right), \quad B_{S}=\frac{1}{2}\left(1-\frac{\varepsilon}{E}\right), \quad g_{S}^{+}=\frac{1}{e^{-E \beta}+1}, \quad g_{S}^{-}=\frac{1}{e^{E \beta}+1} .
$$

$\widehat{\varrho}_{0}$ is the density matrix of noninteracting quasi-particles:

$$
\widehat{\varrho}_{0}=\exp \left\{\frac{\Omega-\sum_{\mathbf{k}, \sigma} E(k) C_{\mathbf{k}, \sigma}^{+} C_{\mathbf{k}, \sigma}}{T}\right\} .
$$

Let us introduce the designations: $-G \Sigma \equiv \Delta F^{+},-G^{+} \Sigma^{+} \equiv \Delta^{+} F$. Then Dyson equation can be rewritten in a form of Gor'kov equations:

$$
\begin{aligned}
& \left(i \omega_{n}-\varepsilon\right) G+\Delta F^{+}=i \\
& \left(i \omega_{n}+\varepsilon\right) F^{+}+G \Delta=0 .
\end{aligned}
$$

The expressions for anomalous propagators follow from Gor'kov equations:

$$
F^{+}\left(\mathbf{k}, \omega_{n}\right)=\frac{-i \Delta^{+}}{\left(i \omega_{n}\right)^{2}-E^{2}(k)}, \quad F\left(\mathbf{k}, \omega_{n}\right)=\left(F^{+}\left(\mathbf{k}, \omega_{n}\right)\right)^{+}=\frac{i \Delta}{\left(i \omega_{n}\right)^{2}-E^{2}(k)} .
$$

We can write the anomalous propagators in $(\mathbf{k}, \mathrm{t})$-space in the form of vacuum averages of creation and annihilation operators:

$$
\begin{aligned}
F_{\alpha \gamma}^{+}(\mathbf{k}, \tau) & =\frac{\Delta^{+}}{\sqrt{\Delta^{+} \Delta}}\left\{\begin{array}{ll}
i \operatorname{Sp}\left\{\widehat{\varrho}_{0} C_{-\mathbf{k}, \gamma}^{+}\left(\tau_{2}\right) C_{\mathbf{k}, \alpha}^{+}\left(\tau_{1}\right)\right\}, & \tau>0 \\
i \operatorname{Sp}\left\{\widehat{\varrho}_{0} C_{\mathbf{k}, \alpha}^{+}\left(\tau_{1}\right) C_{-\mathbf{k}, \gamma}^{+}\left(\tau_{2}\right)\right\}, & \tau \leq 0
\end{array}\right\} \\
& =i g_{\alpha \gamma} \frac{\Delta^{+}}{\sqrt{\Delta^{+} \Delta}} \sqrt{A_{S} B_{S}}\left[\left(g_{S}^{+} e^{-E \tau}-g_{S}^{-} e^{E \tau}\right) \theta_{\tau}-\left(g_{S}^{+} e^{E \tau}-g_{S}^{-} e^{-E \tau}\right) \theta_{-\tau}\right] \\
F_{\alpha \gamma}(\mathbf{k}, \tau) & =\frac{\Delta}{\sqrt{\Delta^{+} \Delta}}\left\{\begin{array}{ll}
-i \operatorname{Sp}\left\{\widehat{\varrho}_{0} C_{\mathbf{k}, \alpha}\left(\tau_{2}\right) C_{-\mathbf{k}, \gamma}\left(\tau_{1}\right)\right\}, & \tau>0 \\
-i \operatorname{Sp}\left\{\widehat{\varrho}_{0} C_{-\mathbf{k}, \gamma}\left(\tau_{1}\right) C_{\mathbf{k}, \alpha}\left(\tau_{2}\right)\right\}, & \tau \leq 0
\end{array}\right\} \\
& =i g_{\alpha \gamma} \frac{\Delta}{\sqrt{\Delta^{+} \Delta}} \sqrt{A_{S} B_{S}}\left[-\left(g_{S}^{+} e^{-E \tau}-g_{S}^{-} e^{E \tau}\right) \theta_{\tau}+\left(g_{S}^{+} e^{E \tau}-g_{S}^{-} e^{-E \tau}\right) \theta_{-\tau}\right] .
\end{aligned}
$$

we can see, that the existence of nonzero anomalous propagators $F$ and $F^{+}$means breakdown of global gauge symmetry in a system, that is the number of particles is not conserved in the course of existence of a pair condensate.

\section{B. Kinetic energy and entropy.}

In order to calculate the free energy it is necessary to know the kinetic energy of system particles, the energy of their interaction and the entropy of collective excitations. The average kinetic energy of all particles of a system is

$$
\langle W\rangle=-2 i \sum_{\mathbf{k}} G\left(\mathbf{k}, \tau \rightarrow 0^{-}\right) \varepsilon(k)=-\frac{2 i}{\beta} \lim _{\tau \rightarrow 0^{-}} \sum_{\mathbf{k}} \sum_{n=-\infty}^{n=+\infty} G\left(\mathbf{k}, \omega_{n}\right) e^{-i \omega_{n} t} \varepsilon(k)
$$




$$
=2 \sum_{\mathbf{k}}\left(g^{-} A+g^{+} B\right) \varepsilon(k)=V 2 \nu_{F} \int_{-v_{F} k_{F}}^{\infty}\left(g^{-} A+g^{+} B\right) \varepsilon d \varepsilon,
$$

where $\nu_{F}=\frac{k_{F}^{2}}{2 \pi^{2} v_{F}}$ is the density of states on Fermy surface. Since the interaction (12) exists only in the layer $-\omega_{D}<\varepsilon(k)<\omega_{D}$, we can suppose that

$$
g^{-}=\left[\begin{array}{cc}
g_{0}^{-} ; & |\varepsilon(k)|>\omega_{D} \\
g_{S}^{-} ; & |\varepsilon(k)|<\omega_{D}
\end{array}\right], \quad g^{+}=\left[\begin{array}{cc}
g_{0}^{+} ; & |\varepsilon(k)|>\omega_{D} \\
g_{S}^{+} ; & |\varepsilon(k)|<\omega_{D}
\end{array}\right] .
$$

Hence, one may write the expression for kinetic energy that separats normal and superconductive parts as

$$
\langle W\rangle=W_{n}+V \nu_{F} \int_{-\omega_{D}}^{\omega_{D}} \tanh \left(\frac{\beta|\varepsilon|}{2}\right) \frac{\varepsilon^{2}}{|\varepsilon|} d \varepsilon-V \nu_{F} \int_{-\omega_{D}}^{\omega_{D}} \tanh \left(\frac{\beta E}{2}\right) \frac{\varepsilon^{2}}{E} d \varepsilon .
$$

If we suppose that $\Delta=0$, then we shall have $W=W_{n}$.

At temperature $T \neq 0$ a gas of collective excitation exists - boholons with the spectrum $E=\sqrt{\varepsilon^{2}(k)+\Delta^{2}}$. Since boholons are product of decay of Cooper pairs to fermions, hence the occupation numbers of states by boholons are

$$
f_{S}(k)=\frac{1}{e^{\beta E}+1}=\frac{1}{2}\left(1-\tanh \left(\frac{\beta E}{2}\right)\right) .
$$

Then the entropy of a system is

$$
\begin{aligned}
S & =-2 \sum_{\mathbf{k}}[f(k) \ln f(k)+(1-f(k)) \ln (1-f(k))] \\
& =S_{0}-2 V \nu_{F} \int_{-\omega_{D}}^{\omega_{D}}\left[f_{S} \ln f_{S}+\left(1-f_{S}\right) \ln \left(1-f_{S}\right)\right] d \varepsilon+2 V \nu_{F} \int_{-\omega_{D}}^{\omega_{D}}\left[f_{0} \ln f_{0}+\left(1-f_{0}\right) \ln \left(1-f_{0}\right)\right] d \varepsilon .
\end{aligned}
$$

Here we separated the normal part again, where $f_{0}=\left(e^{\beta|\varepsilon|}+1\right)^{-1}$, so that $S=S_{n}$ at $\Delta=0$. The multiplier "2" appeared as result of summation over spin states.

\section{Vacuum amplitude.}

In the previous subsections we considered the interaction of particles with fluctuations of pairing, and we found, that the state of a system described by the density matrix $\widehat{\varrho}_{0}$ has another symmetry in comparison with the initial state $\widehat{\rho}_{0}$. The effective Hamiltonian of the system of quasiparticle is

$$
\widehat{H}_{0}+\widehat{H}_{I}=\sum_{\alpha} \sum_{\mathbf{k}} E(\mathbf{k}) C_{\mathbf{k}, \alpha}^{+} C_{\mathbf{k}, \alpha}+\frac{1}{2 V} \sum_{\alpha, \gamma} \sum_{\mathbf{k}, \mathbf{l}} V_{\mathbf{l},-\mathbf{l}, \mathbf{k},-\mathbf{k}} g_{\alpha \gamma} C_{-\mathbf{l}, \gamma}^{+} C_{\mathbf{l}, \alpha}^{+} C_{\mathbf{k}, \alpha} C_{-\mathbf{k}, \gamma}
$$

The vacuum amplitude of a system can be written in the form:

$$
R(\beta)=\langle\widehat{U}(\beta)\rangle_{0}=\operatorname{Sp}\left(\widehat{\varrho}_{0} \widetilde{U}(\beta)\right)=\sum_{n=0}^{\infty} \frac{(-1)^{n}}{n !} \int_{0}^{\beta} d \tau_{1} \ldots \int_{0}^{\beta} d \tau_{n} S p\left(\widehat{\varrho}_{0} T\left\{\widehat{H}_{1}\left(\tau_{I}\right) \ldots \widehat{H}_{I}\left(\tau_{n}\right)\right\}\right),
$$

where $\widehat{H}_{I}(\tau)=e^{+\tau \hat{H}_{0}} \widehat{H}_{I} e^{-\tau \hat{H}_{0}}$ is the interaction operator of particles in interaction representation. The averaging $\operatorname{Sp}\left(\widehat{\varrho}_{0} \widetilde{U}(\beta)\right)$ is done over ensemble of noninteracting quasi-particles. We can write the expended expression for the vacuum amplitude:

$$
\begin{aligned}
& R(\beta)=1+\frac{1}{1 !} \frac{1}{V} \int_{0}^{\beta} d \tau_{1} \sum_{\alpha, \gamma} \sum_{\mathbf{k}, \mathbf{l}}\left(-\frac{1}{2} V_{\mathbf{l},-\mathbf{l}, \mathbf{k},-\mathbf{k}}\right) \operatorname{Sp}\left\{\widehat{\varrho}_{0} C_{-\mathbf{l}, \gamma}^{+}\left(\tau_{1}\right) C_{\mathbf{l}, \alpha}^{+}\left(\tau_{1}\right) C_{\mathbf{k}, \alpha}\left(\tau_{1}\right) C_{-\mathbf{k}, \gamma}\left(\tau_{1}\right)\right\} \\
& +\frac{1}{2 !} \frac{1}{V^{2}} \int_{0}^{\beta} d \tau_{2} \int_{0}^{\beta} d \tau_{1} \sum_{\alpha, \gamma} \sum_{\mathbf{k}, \mathbf{l}}\left(-\frac{1}{2} V_{\mathbf{l},-\mathbf{l}, \mathbf{k},-\mathbf{k}}\right) \sum_{\alpha^{\prime}, \gamma^{\prime}} \sum_{\mathbf{k}^{\prime}, \mathbf{l}^{\prime}}\left(-\frac{1}{2} V_{\mathbf{l}^{\prime},-\mathbf{l}^{\prime}, \mathbf{k}^{\prime},-\mathbf{k}^{\prime}}\right) \\
& \times \operatorname{Sp}\left\{\widehat{\varrho}_{0} C_{-\mathbf{l}^{\prime}, \gamma^{\prime}}^{+}\left(\tau_{2}\right) C_{\mathbf{l}^{\prime}, \alpha^{\prime}}^{+}\left(\tau_{2}\right) C_{\mathbf{k}^{\prime}, \alpha^{\prime}}\left(\tau_{2}\right) C_{-\mathbf{k}^{\prime}, \gamma^{\prime}}\left(\tau_{2}\right) C_{-\mathbf{l}, \gamma}^{+}\left(\tau_{1}\right) C_{\mathbf{l}, \alpha}^{+}\left(\tau_{1}\right) C_{\mathbf{k}, \alpha}\left(\tau_{1}\right) C_{-\mathbf{k}, \gamma}\left(\tau_{1}\right)\right\}+\ldots,
\end{aligned}
$$


where we took into account that $\operatorname{Sp}\left\{\widehat{\varrho}_{0}\right\}=1$. In order to calculate (30) approximately we can uncouple correlations by the following way taking into account anticommutation of operators $C$ and $C^{+}$:

$$
\begin{aligned}
& R(\beta) \approx 1+\frac{1}{1 !}(-1)^{2} \frac{1}{V} \int_{0}^{\beta} d \tau_{1} \sum_{\alpha, \gamma} \sum_{\mathbf{k}, \mathbf{l}}\left(-\frac{1}{2} V_{\mathbf{l},-\mathbf{l}, \mathbf{k},-\mathbf{k}}\right) \operatorname{Sp}\left\{\widehat{\varrho}_{0} C_{\mathbf{l}, \alpha}^{+}\left(\tau_{1}\right) C_{-\mathbf{l}, \gamma}^{+}\left(\tau_{1}\right)\right\} \operatorname{Sp}\left\{\widehat{\varrho}_{0} C_{-\mathbf{k}, \gamma}\left(\tau_{1}\right) C_{\mathbf{k}, \alpha}\left(\tau_{1}\right)\right\} \\
& +\frac{1}{2 !}(-1)^{4} \frac{1}{V^{2}} \int_{0}^{\beta} d \tau_{2} \int_{0}^{\beta} d \tau_{1} \sum_{\alpha, \gamma} \sum_{\mathbf{k}, \mathbf{l}}\left(-\frac{1}{2} V_{\mathbf{l},-\mathbf{l}, \mathbf{k},-\mathbf{k}}\right) \sum_{\alpha^{\prime}, \gamma^{\prime}} \sum_{\mathbf{k}^{\prime}, \mathbf{l}^{\prime}}\left(-\frac{1}{2} V_{\mathbf{l}^{\prime},-\mathbf{l}^{\prime}, \mathbf{k}^{\prime},-\mathbf{k}^{\prime}}\right) \\
& \times \operatorname{Sp}\left\{\widehat{\varrho}_{0} C_{\mathbf{l}^{\prime}, \alpha^{\prime}}^{+}\left(\tau_{2}\right) C_{-\mathbf{l}^{\prime}, \gamma^{\prime}}^{+}\left(\tau_{2}\right)\right\} \operatorname{Sp}\left\{\widehat{\varrho}_{0} C_{-\mathbf{k}^{\prime}, \gamma^{\prime}}\left(\tau_{2}\right) C_{\mathbf{k}^{\prime}, \alpha^{\prime}}\left(\tau_{2}\right)\right\} \operatorname{Sp}\left\{\widehat{\varrho}_{0} C_{\mathbf{l}_{, \alpha}}^{+}\left(\tau_{1}\right) C_{-\mathbf{l}, \gamma}^{+}\left(\tau_{1}\right)\right\} \operatorname{Sp}\left\{\widehat{\varrho}_{0} C_{-\mathbf{k}, \gamma}\left(\tau_{1}\right) C_{\mathbf{k}, \alpha}\left(\tau_{1}\right)\right\} \\
& +\ldots=1+R_{1}+\frac{1}{2 !} R_{1}^{2}+\ldots=\exp \left(R_{1}\right)
\end{aligned}
$$

Let us take into account that our approximation is analogous to Fock approximation for normal processes. A decay of quasi-particles is absent in Hartree-Fock approximation, hence the amplitude of pairing is real $\Delta=\Delta^{+}$in the momentum space. As a consequence we have $F=-F^{+}$. Then $R(t)$ can be written as

$$
\begin{aligned}
& \ln R(\beta)=R_{1}(\beta)=\frac{1}{V} \int_{0}^{\beta} d \tau_{1} \sum_{\alpha, \gamma} \sum_{\mathbf{k}, \mathbf{l}}\left(-\frac{1}{2} V_{\mathbf{l},-\mathbf{l}, \mathbf{k},-\mathbf{k}}\right) i F_{\alpha \gamma}\left(\mathbf{l}, \tau_{1}-\tau_{1}\right) i F_{\alpha \beta}\left(\mathbf{k}, \tau_{1}-\tau_{1}\right) \\
& =\frac{2}{V} \sum_{\mathbf{k}, \mathbf{l}}\left(\frac{1}{2} V_{\mathbf{l},-\mathbf{l}, \mathbf{k},-\mathbf{k}}\right) F\left(\mathbf{l}, \tau \rightarrow 0^{-}\right) F\left(\mathbf{k}, \tau \rightarrow 0^{-}\right) \beta \\
& =\frac{\beta \lambda}{V} \sum_{\mathbf{k}} w_{k} \frac{1}{\beta} \sum_{n=-\infty}^{n=+\infty} F\left(\mathbf{k}, \omega_{n}\right) \sum_{\mathbf{k}} w_{k} \frac{1}{\beta} \sum_{n=-\infty}^{n=+\infty} F\left(\mathbf{k}, \omega_{n}\right) \\
& =-\beta \lambda V \nu_{F}^{2} \int_{-\omega_{D}}^{\omega_{D}} \tanh \left(\frac{\beta E}{2}\right) \frac{\Delta}{2 E} d \varepsilon \int_{-\omega_{D}}^{\omega_{D}} \tanh \left(\frac{\beta E}{2}\right) \frac{\Delta}{2 E} d \varepsilon .
\end{aligned}
$$

The procedure of uncoupling of correlations and the contribution of the anomalous process in the vacuum amplitude

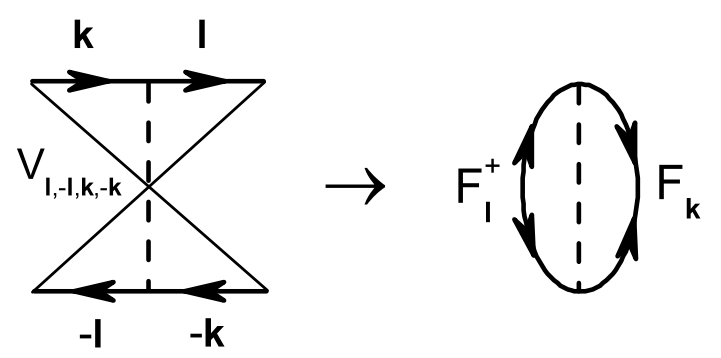

Figure 2: The uncoupling of correlations in the vacuum amplitude for the process of scattering of two fermions by the interaction potential $V_{\mathbf{l},-\mathbf{l}, \mathbf{k},-\mathbf{k}}$ from the initial states $\mathbf{k}, \alpha$ and $-\mathbf{k}, \gamma$ to the final states $\mathbf{l}, \alpha$ and $-\mathbf{l}, \gamma$. As a result we have the anomalous transition amplitude "vacuum-vacuum".

can be represented graphically (Fig 2). In order to calculate the contribution of interaction into free energy we can use the formula (20):

$$
\begin{aligned}
\Omega_{\lambda} & =-\frac{1}{\beta} \ln R(\beta)=-\frac{\lambda}{V} \sum_{\mathbf{k}} w_{k} \frac{1}{\beta} \sum_{n=-\infty}^{n=+\infty} F\left(\mathbf{k}, \omega_{n}\right) \sum_{\mathbf{k}} w_{k} \frac{1}{\beta} \sum_{n=-\infty}^{n=+\infty} F\left(\mathbf{k}, \omega_{n}\right) \\
& =\lambda V \nu_{F}^{2} \int_{-\omega_{D}}^{\omega_{D}} \tanh \left(\frac{\beta E}{2}\right) \frac{\Delta}{2 E} d \varepsilon \int_{-\omega_{D}}^{\omega_{D}} \tanh \left(\frac{\beta E}{2}\right) \frac{\Delta}{2 E} d \varepsilon .
\end{aligned}
$$

If we suppose that $\Delta=0$, then we shall have $\Omega_{\lambda}=0$. 


\section{Free energy.}

Starting from the above found results we can write the expression for free energy of a system:

$$
\begin{aligned}
& \Omega=\langle W\rangle-\frac{1}{\beta} S+\Omega_{\lambda}=-\frac{2 i}{\beta} \lim _{\tau \rightarrow 0^{-}} \sum_{\mathbf{k}} \sum_{n=-\infty}^{n=+\infty} G\left(\mathbf{k}, \omega_{n}\right) e^{-i \omega_{n} t} \varepsilon(k) \\
& +\frac{2}{\beta} \sum_{\mathbf{k}}[f(k) \ln f(k)+(1-f(k)) \ln (1-f(k))]-\frac{\lambda}{V} \sum_{\mathbf{k}} w_{k} \frac{1}{\beta} \sum_{n=-\infty}^{n=+\infty} F\left(\mathbf{k}, \omega_{n}\right) \sum_{\mathbf{k}} w_{k} \frac{1}{\beta} \sum_{n=-\infty}^{n=+\infty} F\left(\mathbf{k}, \omega_{n}\right)
\end{aligned}
$$

The observed value of $\Delta$ must minimize the free energy:

$$
\frac{d \Omega}{d \Delta}=0 \Longrightarrow(-i) \Delta=\frac{\lambda}{V \beta} \sum_{\mathbf{k}} \sum_{n=-\infty}^{n=+\infty} w_{k} F\left(\mathbf{k}, \omega_{n}\right) .
$$

The functional (34) can be written in quadratures:

$$
\begin{aligned}
\Omega= & \Omega_{n}+V \nu_{F} \int_{-\omega_{D}}^{\omega_{D}}\left[\tanh \left(\frac{\beta|\varepsilon|}{2}\right) \frac{\varepsilon^{2}}{|\varepsilon|}-\tanh \left(\frac{\beta E}{2}\right) \frac{\varepsilon^{2}}{E}\right] d \varepsilon \\
& +\frac{2 V}{\beta} \nu_{F} \int_{-\omega_{D}}^{\omega_{D}}\left[f_{S} \ln f_{S}+\left(1-f_{S}\right) \ln \left(1-f_{S}\right)-f_{0} \ln f_{0}-\left(1-f_{0}\right) \ln \left(1-f_{0}\right)\right] d \varepsilon \\
& +V \nu_{F} g \int_{-\omega_{D}}^{\omega_{D}} \tanh \left(\frac{\beta E}{2}\right) \frac{\Delta}{2 E} d \varepsilon \int_{-\omega_{D}}^{\omega_{D}} \tanh \left(\frac{\beta E}{2}\right) \frac{\Delta}{2 E} d \varepsilon,
\end{aligned}
$$

where $\Omega_{n}$ is the energy of a normal phase, $g \equiv \lambda \nu_{F}$ is the effective interaction constant, $V$ is the volume of a system. We have $\Omega=\Omega_{n}$ for the case $\Delta=0$. The equilibrium value of $\Delta$ is determined by balance of kinetic energy, the entropy term and the energy of interaction. The parameter $g$ can be expressed via critical temperature $\beta_{C}$ with help of $\Delta\left(\beta_{C}\right)=0$ as following:

$$
1=-g \int_{-\omega_{D}}^{\omega_{D}} \tanh \left(\frac{\beta_{C}|\varepsilon|}{2}\right) \frac{1}{2|\varepsilon|} d \varepsilon
$$

Let us consider a low-temperature limit of the free energy (36): $\Delta \beta \gg 1$ at $T \rightarrow 0$. This means, that the value $\Delta-\Delta_{0}$ can be chosen as the expansion parameter, where $\Delta_{0}=\Delta(T=0)$ is the equilibrium value of gap (amplitude of pairing) at zero temperature. Then the low-temperature expansion has a form:

$$
\Omega=\Omega_{n}+V\left(\alpha_{0}(T)+b_{0}(T) \Delta+d_{0} \Delta^{2}\right),
$$

where the expansion coefficients in the approximation $g \ll 1 \Rightarrow \Delta \ll \omega_{D}$ are

$$
\begin{aligned}
& \alpha_{0}(T)=\frac{\nu_{F}}{2} \Delta_{0}^{2}+4 \nu_{F} T^{2}-\nu_{F} \sqrt{8 \pi \Delta_{0} T^{3}} e^{-\frac{\Delta_{0}}{T}}-\nu_{F} \sqrt{8 \pi \Delta_{0}^{3} T} e^{-\frac{\Delta_{0}}{T}} \\
& b_{0}(T)=-2 \nu_{F} \Delta_{0}+\nu_{F} \sqrt{8 \pi \Delta_{0} T} e^{-\frac{\Delta_{0}}{T}}, \quad d_{0}=\nu_{F}
\end{aligned}
$$

The equilibrium value of the energy gap is $\Delta(T)=\frac{b_{0}(T)}{2 d_{0}}=\Delta_{0}\left(1-\sqrt{\frac{2 \pi T}{\Delta_{0}}} e^{-\frac{\Delta_{0}}{T}}\right)$.

Let us consider a high-temperature limit of the free energy: $\Delta \beta_{C} \ll 1$ at $T \rightarrow T_{C}$. Due to a rapid convergence of integration elements in (36), the limits of integration can be $-\infty,+\infty$. Then the expansion in powers of $\Delta$ gives:

$$
\Omega=\Omega_{n}+V\left(\alpha(T) \Delta^{2}+\frac{1}{2} b \Delta^{4}+\frac{1}{3} d \Delta^{6}\right),
$$

where the coefficients of the expansion are

$$
\begin{aligned}
\alpha(T) & =\nu_{F} \frac{T-T_{c}}{T_{c}}, \quad b=\nu_{F} \frac{7 \zeta(3)}{8 \pi^{2} T_{c}^{2}} \\
d & =\nu_{F}\left(\frac{52.31 \zeta(5)}{\pi^{4}}+4.83\right) \frac{1}{4 ! T_{c}^{4}} .
\end{aligned}
$$

This expansion has a form of Landau expansion of free energy in powers of the order parameter. 


\section{FREE ENERGY OF SPATIALLY INHOMOGENEOUS SUPERCONDUCTOR.}

In the previous sections we supposed, that amplitudes of pairing $\Delta$ and $\Delta^{+}$do not depend on spatial coordinates. This takes place in interminable, homogeneous, isotropic and isolated from external fields superconductor. However in a general case these conditions are not realized. For example, in a sufficiently strong magnetic field the inclusions of normal phase can exist in volume of a superconductor. Another example is the contact of a superconductor and a normal metal. In this case the order parameter is suppressed in the boundary layer of a superconductor, however it appears in the boundary layer of a normal metal. We understand a spatially inhomogeneous superconductor as a superconductor with spatially inhomogeneous distribution of the order parameter: $\Delta(\mathbf{r})$ and $\Delta^{+}(\mathbf{r})$.

In the previous sections we considered the pairing of fermions with the opposite momentums: $\mathbf{k}$ and $-\mathbf{k}$. Now let fermions can pair with arbitrary momentums: $\mathbf{k}+\mathbf{q}$ and $-\mathbf{k}$, where $\mathbf{q}$ passes through all the vector space. The potential of particles' interaction in the given states is $V_{\mathbf{l}+\mathbf{q},-\mathbf{l}, \mathbf{k}+\mathbf{q},-\mathbf{k}}=\lambda w_{k} w_{l}, \quad \lambda<0$. For convenience let us pass to the reference system of center of mass of a pair, where the momentums of particles are equal by modulus and are directed oppositely:

$$
\begin{aligned}
& \mathbf{p}_{01}=\frac{m_{2} \mathbf{p}_{1}-m_{1} \mathbf{p}_{2}}{m_{1} m_{2}}=\mathbf{k}+\frac{\mathbf{q}}{2} \\
& \mathbf{p}_{01}=-\frac{m_{2} \mathbf{p}_{1}-m_{1} \mathbf{p}_{2}}{m_{1} m_{2}}=-\mathbf{k}-\frac{\mathbf{q}}{2}
\end{aligned}
$$

because $m_{1}=m_{2}=m$. Hence the system hamiltonian has the form:

$$
\begin{aligned}
\widehat{H}_{\mathrm{eff}} & =\sum_{\mathbf{q}} \sum_{\alpha} \sum_{\mathbf{k}} \varepsilon\left(\mathbf{k}+\frac{\mathbf{q}}{2}\right) C_{\mathbf{k}+\frac{\mathbf{q}}{2}, \alpha}^{+} C_{\mathbf{k}+\frac{\mathbf{q}}{2}, \alpha} \\
& +\frac{1}{2 V} \sum_{\mathbf{q}} \sum_{\alpha, \gamma} \sum_{\mathbf{k}, \mathbf{l}} \lambda w_{k} w_{l} g_{\alpha \gamma} C_{-\mathbf{l}-\frac{\mathbf{q}}{2}, \gamma}^{+} C_{\mathbf{l}+\frac{\mathbf{q}}{2}, \alpha}^{+} C_{\mathbf{k}+\frac{\mathbf{q}}{2}, \alpha} C_{-\mathbf{k}-\frac{\mathbf{q}}{2}, \gamma} .
\end{aligned}
$$

In the system with a such hamiltonian the Cooper instability takes place either as for the system with the hamiltonian (11). However now the amplitude of pairing is function of $\mathbf{q}: \Delta \rightarrow \Delta \mathbf{q}$. If the energy gap is function of a wave vector $\Delta=\Delta(\mathbf{q})$ then the energy gap is function of a radius-vector: $\Delta=\Delta(\mathbf{r})$, because the Fourier transformation realizes the one to one correspondence between functions defined in the $\mathbf{q}$-space and in the $\mathbf{r}$-space. Taking into account the reality of the order parameter in the momentum space $\Delta_{\mathbf{q}}=\Delta_{\mathbf{q}}^{+}$and requiring identical dimension of the order parameter in the $\mathbf{q}$-space and in the $\mathbf{r}$-space we can write the Fourier-transformations as:

$$
\begin{aligned}
\Delta(\mathbf{r}) & =\sum_{\mathbf{q}} \Delta(\mathbf{q}) e^{i \mathbf{q r}}=\frac{V}{(2 \pi)^{3}} \int \Delta(\mathbf{q}) e^{i \mathbf{q} \mathbf{r}} d^{3} q \\
\Delta^{+}(\mathbf{r}) & =\sum_{\mathbf{q}} \Delta(q) e^{-i \mathbf{q r}}=\frac{V}{(2 \pi)^{3}} \int \Delta(\mathbf{q}) e^{-i \mathbf{q} \mathbf{r}} d^{3} q, \\
\Delta(\mathbf{q}) & =\frac{1}{V} \int \Delta(\mathbf{r}) e^{-i \mathbf{q} \mathbf{r}} d^{3} r=\frac{1}{V} \int \Delta^{+}(\mathbf{r}) e^{i \mathbf{q r}} d^{3} r \\
-\sum_{\mathbf{q}} & =\frac{\mathbf{k}+\mathbf{q} / 2}{-\Delta_{\mathbf{q}}}{ }_{\mathrm{iG}_{0}^{+}\left(-\mathbf{k}-\mathbf{q} / 2, \omega_{\mathrm{n}}\right)}^{-\mathbf{k}-\mathbf{q} / 2}
\end{aligned}
$$

Figure 3: The diagram for the mass operator $\Sigma$ describing an interaction of a fermion with fluctuations of pairing in the spatially inhomogeneous system.

The mass operator for the process of interaction of a particle with the fluctuations of pairing is shown in Fig 3 . In analytical representation it has a view:

$$
-\Sigma_{\mathbf{q}}\left(\mathbf{k}, \omega_{n}\right)=\left(-\Delta_{\mathbf{q}}\right) i G_{0}^{+}\left(-\mathbf{k}-\frac{\mathbf{q}}{2}, \omega_{n}\right)\left(-\Delta_{\mathbf{q}}^{+}\right)=\frac{-\Delta_{\mathbf{q}} \Delta_{\mathbf{q}}^{+}}{i \omega_{n}+\varepsilon\left(\mathbf{k}+\frac{\mathbf{q}}{2}\right)},
$$


where the free propagator $G_{0}$ is

$$
G_{0}=\frac{1}{i \omega_{n}-\varepsilon\left(\mathbf{k}+\frac{\mathbf{q}}{2}\right)}=i \frac{i \omega_{n}+\varepsilon_{\mathbf{q}}(k)}{\left(i \omega_{n}\right)^{2}-\varepsilon_{\mathbf{q}}^{2}(k)}
$$

Then from Dyson equation we can obtain the dressed propagator:

$$
\frac{1}{G_{0}}=\frac{1}{G_{S}}-i \Sigma_{\mathbf{q}} \Rightarrow G_{S}=i \frac{i \omega_{n}+\varepsilon_{\mathbf{q}}}{\left(i \omega_{n}\right)^{2}-E_{\mathbf{q}}^{2}}
$$

where $E_{\mathbf{q}}$ is the specter of quasi-particles in a inhomogeneous system:

$$
E_{\mathbf{q}}^{2}=\varepsilon_{\mathbf{q}}^{2}+\left|\Delta_{\mathbf{q}}\right|^{2}, \quad \varepsilon_{\mathbf{q}} \equiv \varepsilon\left(\mathbf{k}+\frac{\mathbf{q}}{2}\right) \approx \varepsilon(k)+\frac{\mathbf{k q}}{2 m}, \quad|\mathbf{k}| \simeq k_{F}, \quad|\mathbf{q}| \ll k_{F} .
$$

Dyson equation can be represented in a form of Gor'kov equations set, from where the expressions for anomalous propagators follow:

$$
\begin{gathered}
\left(i \omega_{n}-\varepsilon_{\mathbf{q}}\right) G+\Delta_{\mathbf{q}} F^{+}=i \\
\left(i \omega_{n}+\varepsilon_{\mathbf{q}}\right) F^{+}+G \Delta_{\mathbf{q}}=0
\end{gathered} \Rightarrow \begin{gathered}
F_{\mathbf{q}}^{+}\left(\mathbf{k}, \omega_{n}\right)=\frac{-i \Delta_{\mathbf{q}}^{+}}{\left(i \omega_{n}\right)^{2}-E_{\mathbf{q}}^{2}} \\
F_{\mathbf{q}}\left(\mathbf{k}, \omega_{n}\right)=\left(F_{\mathbf{q}}^{+}\left(\mathbf{k}, \omega_{n}\right)\right)^{+}=\frac{i \Delta_{\mathbf{q}}}{\left(i \omega_{n}\right)^{2}-E_{\mathbf{q}}^{2}}
\end{gathered}
$$

If to suppose $q=0$, then we shall have the expressions (18, 19).

Now let us suppose that the order parameter $\Delta(\mathbf{r})$ changes very slowly on a coherence length $l(T)$ which characterizes a size of Cooper pair (the long-wave approximation $q \rightarrow 0, q l(T) \ll 1$ ). Then we $\operatorname{can} \operatorname{suppose} \varepsilon\left(\mathbf{k}+\frac{\mathbf{q}}{2}\right) \approx \varepsilon(\mathbf{k})$ in the specter of quasi-particles, such that $E_{\mathbf{q}} \approx \sqrt{\varepsilon^{2}(k)+\left|\Delta_{\mathbf{q}}\right|^{2}}$. However we must keep $\varepsilon\left(\mathbf{k}+\frac{\mathbf{q}}{2}\right)$ in numerators of the expressions (46) and (47) for $G$. Hence the normal propagator has a form:

$G_{\mathbf{q}}(\mathbf{k}, \tau)=-i \theta_{\tau}\left(g_{\mathbf{q}}^{+} A\left(\mathbf{k}+\frac{\mathbf{q}}{2}\right) e^{-E_{\mathbf{q}} \tau}+g_{\mathbf{q}}^{-} B\left(\mathbf{k}+\frac{\mathbf{q}}{2}\right) e^{E} \mathbf{q}^{\tau}\right)+i \theta_{-\tau}\left(g_{\overline{\mathbf{q}}}^{-} A\left(\mathbf{k}+\frac{\mathbf{q}}{2}\right) e^{-E_{\mathbf{q}} \tau}+g_{\mathbf{q}}^{+} B\left(\mathbf{k}+\frac{\mathbf{q}}{2}\right) e^{E} \mathbf{q}^{\tau}\right)(5$

where

$$
A\left(\mathbf{k}+\frac{\mathbf{q}}{2}\right) \approx A_{\mathbf{q}}(k)+\frac{1}{2 E_{\mathbf{q}}} \frac{\mathbf{k q}}{2 m}, \quad B\left(\mathbf{k}+\frac{\mathbf{q}}{2}\right) \approx B_{\mathbf{q}}(k)-\frac{1}{2 E_{\mathbf{q}}} \frac{\mathbf{k q}}{2 m} .
$$

The anomalous propagators are

$$
\begin{aligned}
& F_{\alpha \gamma}^{+}(\mathbf{k}, \mathbf{q}, \tau)=i g_{\alpha \beta} \frac{\Delta_{\mathbf{q}}^{+}}{2 E_{\mathbf{q}}}\left[\left(g_{\mathbf{q}}^{+} e^{-E_{\mathbf{q}^{\tau}}}-g_{\mathbf{q}} e^{E \mathbf{q}^{\tau}}\right) \theta_{\tau}-\left(g_{\mathbf{q}}^{+} e^{E_{\mathbf{q}} \tau}-g_{\mathbf{q}} e^{-E_{\mathbf{q}} \tau}\right) \theta_{-\tau}\right] \\
& F_{\alpha \gamma}(\mathbf{k}, \mathbf{q}, \tau)=i g_{\alpha \beta} \frac{\Delta_{\mathbf{q}}}{2 E_{\mathbf{q}}}\left[-\left(g_{\mathbf{q}}^{+} e^{-E_{\mathbf{q}} \tau}-g_{\mathbf{q}}^{-} e^{E} \mathbf{q}^{\tau}\right) \theta_{\tau}+\left(g_{\mathbf{q}}^{+} e^{E} \mathbf{q}^{\tau}-g_{\mathbf{q}}^{-} e^{-E_{\mathbf{q}} \tau}\right) \theta_{-\tau}\right] .
\end{aligned}
$$

We can see, that in the long wave approximation the anomalous propagators depend on $\mathbf{q}$ by means of $\Delta(\mathbf{q})$ only.

Kinetic energy of a system is determined by the following way:

$$
\begin{aligned}
\langle W\rangle & =-2 i \sum_{\mathbf{q}} \sum_{\mathbf{k}} \varepsilon\left(\mathbf{k}+\frac{\mathbf{q}}{2}\right) G\left(\mathbf{k}+\frac{\mathbf{q}}{2}, \tau \rightarrow 0^{-}\right) \\
& =W_{n}+V \nu_{F} \sum_{\mathbf{q}} \int_{-\omega_{D}}^{\omega_{D}}\left[\tanh \left(\frac{\beta|\varepsilon|}{2}\right) \frac{\varepsilon^{2}}{|\varepsilon|}-\tanh \left(\frac{\beta E_{\mathbf{q}}}{2}\right) \frac{\varepsilon^{2}}{E_{\mathbf{q}}}\right] d \varepsilon \\
& +V \nu_{F} \frac{v_{F}^{2}}{12} \sum_{\mathbf{q}} \int_{-\omega_{D}}^{\omega_{D}} \mathbf{q}^{2}\left[\tanh \left(\frac{\beta|\varepsilon|}{2}\right) \frac{1}{|\varepsilon|}-\tanh \left(\frac{\beta E_{\mathbf{q}}}{2}\right) \frac{1}{E_{\mathbf{q}}}\right] d \varepsilon
\end{aligned}
$$

We can see, that the term, which is proportional to $\mathbf{q}^{2}$, is added to the kinetic energy (25) (with the replacement $\left.E \rightarrow E_{\mathbf{q}}=\sqrt{\varepsilon^{2}(k)+|\Delta(\mathbf{q})|^{2}}\right)$. In the long wave approximation the expressions for entropy and vacuum amplitude 
coincide with the expressions (27) and (32) accordingly, however it should be written $\Delta(\mathbf{q})$ instead of $\Delta=$ const. Then we can write the free energy:

$$
\Omega=\Omega_{n}+\sum_{\mathbf{q}} \Omega\left(\Delta_{\mathbf{q}}\right)+V \nu_{F} \frac{v_{F}^{2}}{12} \sum_{\mathbf{q}} \int_{-\omega_{D}}^{\omega_{D}} \mathbf{q}^{2}\left[\tanh \left(\frac{\beta|\varepsilon|}{2}\right) \frac{1}{|\varepsilon|}-\tanh \left(\frac{\beta E_{\mathbf{q}}}{2}\right) \frac{1}{E_{\mathbf{q}}}\right] d \varepsilon
$$

where $\Omega\left(\Delta_{\mathbf{q}}\right)$ coincides with the expression (36), where the replacement $\Delta \rightarrow \Delta(\mathbf{q})$ was done.

Expanding the free energy (54) in powers of $\Delta$ we can obtain the expression:

$$
\Omega=\Omega_{n}+V \sum_{\mathbf{q}}\left(\alpha(T) \Delta_{\mathbf{q}}^{2}+\frac{1}{2} b \Delta_{\mathbf{q}}^{4}+\gamma q^{2} \Delta_{\mathbf{q}}^{2}\right)=\Omega_{n}+\frac{V^{2}}{(2 \pi)^{3}} \int\left(\alpha(T) \Delta_{\mathbf{q}}^{2}+\frac{1}{2} b \Delta_{\mathbf{q}}^{4}+\gamma \mathbf{q}^{2} \Delta_{\mathbf{q}}^{2}\right) d^{3} q
$$

where the coefficient $\gamma$ is

$$
\gamma=\nu_{F} \frac{7 \zeta(3) v_{F}^{2}}{48 \pi^{2} T_{c}^{2}}=\nu_{F} l_{0}^{2}
$$

where $l_{0}$ is a coherence length at $T=0$ (Pippard length). The expansion (55) has a form of Landau expansion of free energy in powers of order parameter at the condition $q l_{0} \ll 1$. We can see, that a spatial inhomogeneity increases the free energy of a superconductor. Hence in most cases we can restrict the free energy expansion to the term $\sim q^{2}$, because more fast changes of $\Delta$ increase the free energy essentially.

Let's pass from momentum space to real space using the expressions (44):

$$
\begin{aligned}
& \int \Delta_{\mathbf{q}} \Delta_{\mathbf{q}} d^{3} q=\int \Delta_{\mathbf{q}} {\left[\frac{1}{V} \int \Delta(\mathbf{r}) e^{-i \mathbf{q} \mathbf{r}} d^{3} r\right] d^{3} q=\frac{1}{V} \int \Delta(\mathbf{r})\left[\int \Delta(\mathbf{q}) e^{-i \mathbf{q} \mathbf{r}} d^{3} q\right] d^{3} r=\frac{(2 \pi)^{3}}{V^{2}} \int \Delta(\mathbf{r}) \Delta^{+}(\mathbf{r}) d^{3} r,(57} \\
& \int \mathbf{q} \Delta_{\mathbf{q}} \mathbf{q} \Delta_{\mathbf{q}} d^{3} q=\int \mathbf{q} \Delta_{\mathbf{q}}\left[\frac{1}{V} \int e^{-i \mathbf{q} \mathbf{r}}(-i) \frac{\partial}{\partial \mathbf{r}} \Delta(\mathbf{r}) d^{3} r\right] d^{3} q \\
&\left.=\frac{-i}{V} \int\left[\int \mathbf{q} \Delta(\mathbf{q}) e^{-i \mathbf{q} \mathbf{r}} d^{3} q\right] \frac{\partial}{\partial \mathbf{r}} \Delta(\mathbf{r}) d^{3} r=\frac{(2 \pi)^{3}}{V^{2}} \int \frac{\partial}{\partial \mathbf{r}} \Delta(\mathbf{r})\right] \frac{\partial}{\partial \mathbf{r}} \Delta^{+}(\mathbf{r}) d^{3} r
\end{aligned}
$$

For the term $\Delta^{4}$ and terms with more high powers the situation is more difficult. This is because a square of a Fourier transform is not equal to a Fourier transform of a square: $\left(\frac{1}{V} \int \Delta(\mathbf{r}) e^{-i \mathbf{q} \mathbf{r}} d^{3} r\right)^{2} \neq \frac{1}{V} \int \Delta^{2}(\mathbf{r}) e^{-i \mathbf{q r}} d^{3} r$. Apparently this fact is the manifestation of a nonlocality of the order parameter in zero magnetic field [19]: the value of gap in a point is determined by a distribution of the gap in a some neighborhood of this point: $\Delta(\mathbf{r})=\int d \mathbf{r}^{\prime} Q\left(\mathbf{r}, \mathbf{r}^{\prime}\right) \Delta\left(\mathbf{r}^{\prime}\right)$, where $Q\left(\mathbf{r}, \mathbf{r}^{\prime}\right)$ is the kernel in coordinate space. In a simplest case the kernel $Q$ is the function of the distance $\left|\mathbf{r}-\mathbf{r}^{\prime}\right|$ only. In a total nonlocal case the kernel is function of the gap $\Delta\left(\mathbf{r}^{\prime}\right)$ also. If we neglect of this correlation then we shall obtain the expansion of the free energy in powers of $\Delta \Delta^{+}$in real space in the following form:

$$
\Omega=\Omega_{n}+\int\left[\alpha(T)|\Delta(\mathbf{r})|^{2}+\frac{b}{2}|\Delta(\mathbf{r})|^{4}+\gamma\left|\frac{\partial}{\partial \mathbf{r}} \Delta(\mathbf{r})\right|^{2}\right] d^{3} r
$$

This expansion coincides with Ginzburg-Landau expansion in zero magnetic field. As a result of minimization of the functional (59) $\frac{\delta \Omega}{\delta \Delta}=0$ with a consideration of corresponding boundary conditions we obtain the energy gap as the function of coordinates $\Delta=\Delta(\mathbf{r})$. It prove our statement about that the pairing of fermions with the momentums $\mathbf{k}+\mathbf{q}$ and $-\mathbf{k}$ (or $\mathbf{k}+\frac{\mathbf{q}}{2}$ and $-\mathbf{k}-\frac{\mathbf{q}}{2}$ in a system of center of mass) results to the spatially inhomogeneous order parameter if the wave-vector $\mathbf{q}$ passes through all the vector space. The physical interpretation of this fact lies in the following. Let us assume that we have a superconductor with spatially homogeneous distribution of the order parameter. This distribution corresponds to a minimum of the free energy. Then we shall create an inhomogeneity in the distribution of energy gap by some way. This inhomogeneity increases the free energy according to (54) or (59). This means that we is doing some work on the system. This work is spent on infusion of an additional momentum to each partner of a pair: $\mathbf{k} \rightarrow \mathbf{k}+\frac{\mathbf{q}}{2}$ and $-\mathbf{k} \rightarrow-\mathbf{k}-\frac{\mathbf{q}}{2}$.

Minimization of the functional (55) gives (at the condition $q \ll l(T)$ ):

$$
\Delta_{\mathbf{q}}^{2}=-\frac{\alpha}{b}\left(1+\frac{\gamma}{\alpha} q^{2}\right)=\Delta^{2}(T)\left(1-l^{2}(T) q^{2}\right) \approx \frac{\Delta^{2}(T)}{1+l^{2}(T) q^{2}} \Rightarrow Q(\mathbf{q}) \approx \frac{1}{1+l^{2}(T) q^{2} / 2},
$$


where $Q(\mathbf{q})$ is the kernel in momentum space, $l(T)=l_{0} /|\alpha(T)|$ is the coherent length at temperature $T$. At the critical temperature $T \rightarrow T_{C}$ we have $l\left(T_{C}\right)=\infty$. This means that at high temperatures the nonlocality is caused by both the own nonlocality of a superconductor and by fluctuations in the critical region. We can see that in the long-wave approximation the kernel $Q$ is the function of the distance $\left|\mathbf{r}-\mathbf{r}^{\prime}\right|$ only: $Q\left(\left|\mathbf{r}-\mathbf{r}^{\prime}\right|\right)=\sum_{\mathbf{q}} Q(\mathbf{q}) e^{i \mathbf{q}\left(\mathbf{r}-\mathbf{r}^{\prime}\right)}$.

Let's consider the low-temperature expansion:

$$
\Omega=\Omega_{n}+V \sum_{\mathbf{q}}\left(\alpha_{0}(T)+b_{0}(T) \Delta_{\mathbf{q}}+d_{0} \Delta_{\mathbf{q}}^{2}+\gamma q^{2} \Delta_{\mathbf{q}}^{2}\right)
$$

where coefficients $\alpha_{0}(T), b_{0}(T), d_{0}$ are determined by the formulas (39), and coefficient $\gamma$ is determined by the formula (38). The observed configuration of order parameter $\Delta_{\mathbf{q}}$ minimized the free energy:

$$
\frac{\delta \Omega}{\delta \Delta}=0 \Rightarrow b_{0}(T)+2 d_{0} \Delta_{\mathbf{q}}+\gamma q^{2} \Delta_{\mathbf{q}}=0 \Rightarrow \Delta_{\mathbf{q}}(T)=\frac{-b_{0}(T)}{2 d_{0}+2 \gamma q^{2}}=\frac{\Delta(T)}{1+l_{0}^{2} q^{2} / 2} \Rightarrow Q \approx \frac{1}{1+l_{0}^{2} q^{2} / 2}
$$

Thus the energy gap (the amplitude of pairing) $\Delta_{\mathbf{q}}$ is the nonlocal order parameter with the correlation length $l_{0}$.

The dependence of the interaction constant on coordinates $\lambda(\mathbf{r})=\sum_{\mathbf{q}} \lambda(\mathbf{q}) e^{i \mathbf{q r}}$ is the direct cause of a spatial inhomogeneity. Hamiltonian of such system is

$$
\begin{aligned}
\widehat{H}_{\mathrm{eff}} & =\sum_{\mathbf{q}} \sum_{\alpha} \sum_{\mathbf{k}} \varepsilon_{q}\left(\mathbf{k}+\frac{\mathbf{q}}{2}\right) C_{\mathbf{k}+\frac{\mathbf{q}}{2}, \alpha}^{+} C_{\mathbf{k}+\frac{\mathbf{q}}{2}, \alpha} \\
& +\frac{1}{2 V} \sum_{\mathbf{q}} \sum_{\alpha, \gamma} \sum_{\mathbf{k}, \mathbf{l}} \lambda(\mathbf{q}) w_{k} w_{l} g_{\alpha \gamma} C_{-{ }_{-}-\frac{\mathbf{q}}{2}, \gamma} C_{\mathbf{l}_{+\frac{\mathbf{q}}{2}, \alpha}^{+}} C_{\mathbf{k}+\frac{\mathbf{q}}{2}, \alpha} C_{-\mathbf{k}-\frac{\mathbf{q}}{2}, \gamma},
\end{aligned}
$$

Hence the free energy of a spatially inhomogeneous superconductor has the form:

$$
\begin{aligned}
& \Omega=-\frac{2 i}{\beta} \lim _{\tau \rightarrow 0^{-}} \sum_{\mathbf{q}} \sum_{\mathbf{k}} \sum_{n=-\infty}^{n=+\infty} G_{\mathbf{q}}\left(\mathbf{k}, \omega_{n}\right) e^{-i \omega_{n} \tau} \varepsilon\left(\mathbf{k}+\frac{\mathbf{q}}{2}\right) \\
& +\sum_{\mathbf{q}} \sum_{\mathbf{k}} \frac{2}{\beta}\left[f_{\mathbf{q}}^{S} \ln f_{\mathbf{q}}^{S}+\left(1-f_{\mathbf{q}}^{S}\right) \ln \left(1-f_{\mathbf{q}}^{S}\right)-f_{\mathbf{q}}^{0} \ln f_{\mathbf{q}}^{0}-\left(1-f_{\mathbf{q}}^{0}\right) \ln \left(1-f_{\mathbf{q}, \mathbf{a}}^{0}\right)\right] \\
& -\sum_{\mathbf{q}} \frac{\lambda(\mathbf{q})}{V} \sum_{\mathbf{k}} w_{k} \frac{1}{\beta} \sum_{n=-\infty}^{n=+\infty} F_{\mathbf{q}}\left(\mathbf{k}, \omega_{n}\right) \sum_{\mathbf{k}} w_{k} \frac{1}{\beta} \sum_{n=-\infty}^{n=+\infty} F_{\mathbf{q}}\left(\mathbf{k}, \omega_{n}\right)
\end{aligned}
$$

the observed value of $\Delta_{\mathbf{q}, \mathbf{a}}$ must minimize the free energy:

$$
\frac{d \Omega}{d \Delta_{\mathbf{q}}}=0 \Longrightarrow(-i) \Delta_{\mathbf{q}}=\frac{\lambda(\mathbf{q})}{V \beta} \sum_{\mathbf{k}} \sum_{n=-\infty}^{n=+\infty} w_{k} F_{\mathbf{q}}\left(\mathbf{k}, \omega_{n}\right) \Rightarrow 1=-\frac{\lambda(\mathbf{q})}{V} \sum_{\mathbf{k}} w_{k} \frac{1}{2 E_{\mathbf{q}}} \tanh \left(\frac{\beta E_{\mathbf{q}}}{2}\right) .
$$

The equation (65) generalizes Ginzburg-Landau equation for any temperature and for arbitrary spatial variations of the order parameter. The kernel $Q$ is the function of both the distance $\left|\mathbf{r}-\mathbf{r}^{\prime}\right|$ and the order parameter $\Delta\left(\mathbf{r}^{\prime}\right)$ now. This means, that the fast changing in space order parameter is strongly nonlocal.

In order to write the high-temperature expansion we can introduce the local critical temperature $T_{C}(\mathbf{q})$ with help of the equation (65) assuming $\Delta=0$ and $\varepsilon_{\mathbf{q}}=\varepsilon$ :

$$
1=-g(\mathbf{q}) \int_{-\omega_{D}}^{\omega_{D}} \tanh \left(\frac{\beta_{C}(\mathbf{q})|\varepsilon|}{2}\right) \frac{d \varepsilon}{2|\varepsilon|},
$$

where $g(\mathbf{q}) \equiv \lambda(\mathbf{q}) \nu_{F}$. If $\Delta_{\mathbf{q}} / T \ll 1$ then we can expanse the free energy functional (64) in powers of $\Delta_{\mathbf{q}}$. In the long-wave limit we has the expression:

$$
\Omega=\Omega_{n}+V \sum_{\mathbf{q}}\left(\nu_{F} \frac{T-T_{C}(\mathbf{q})}{T_{C}(\mathbf{q})} \Delta_{\mathbf{q}}^{2}+\frac{\nu_{F}}{2} \frac{7 \zeta(3)}{8 \pi^{2} T_{C}^{2}(\mathbf{q})} \Delta_{\mathbf{q}}^{4}+\nu_{F} \frac{7 \zeta(3) v_{F}^{2}}{48 \pi^{2} T_{C}^{2}(\mathbf{q})} q^{2} \Delta_{\mathbf{q}}^{2}\right) .
$$

Moreover the correlation length can be introduced formally: $\gamma(\mathbf{q})=\nu_{F} l_{0}^{2}(\mathbf{q})$. In the $\mathbf{r}$-space the coefficients of the expansion is functions of coordinates $\alpha(T, \mathbf{r}), b(\mathbf{r}), \gamma(\mathbf{r})$ and they can be obtained by the microscopic way only. 
The value $\Delta_{0}(\mathbf{q})-\Delta_{\mathbf{q}}(T)$ can be the parameter of the low temperature expansion, where the value $\Delta_{0}(\mathbf{q})$ can be obtained from the equation (65) assuming $T=0$ and $\varepsilon_{\mathbf{q}}=\varepsilon$ :

$$
1=-g(\mathbf{q}) \int_{-\omega_{D}}^{\omega_{D}} \frac{d \varepsilon}{2 \sqrt{\varepsilon^{2}+\Delta_{0}^{2}(\mathbf{q})}} \tanh \left(\frac{\beta \sqrt{\varepsilon^{2}+\Delta_{0}^{2}(\mathbf{q})}}{2}\right) .
$$

Then the low-temperature expansion has the form (61) in the long-wave limit, where the coefficients $\alpha_{0}(T, \mathbf{q}), b_{0}(\mathbf{q})(T), d_{0}$ determined by the formulas (39) with the replacement $\Delta_{0} \rightarrow \Delta_{0}(\mathbf{q})$, and coefficient $\gamma$ is determined by the formula (38) with the replacement $l_{0} \rightarrow l_{0}(\mathbf{q})$. In the $\mathbf{r}$-space the coefficients is functions of coordinates $\alpha_{0}(T, \mathbf{r}), b_{0}(\mathbf{r})(T), \gamma(\mathbf{r})$ and they can be obtained by the microscopic way only.

\section{THE FREE ENERGY OF SPATIALLY INHOMOGENEOUS SUPERCONDUCTOR IN A MAGNETIC FIELD.}

In this section we shall generalize the previous results for the case, when a superconductor is placed in magnetic field $\mathbf{H}(\mathbf{r})=\operatorname{rot} \mathbf{A}(r)$. Our aim is to obtain the functional of free energy $\Omega\left(\Delta(\mathbf{r}), \frac{\partial}{\partial \mathbf{r}} \Delta(\mathbf{r})\right.$, $\left.\mathbf{A}(\mathbf{r})\right)$, which is correct for arbitrary value of the relation $\Delta(T) / T$, for arbitrary spatial variations of the order parameter $\Delta(\mathbf{r})$, for an arbitrary value of a magnetic penetration depth $\lambda(T)$ in comparison with a coherent length $l_{0}$ (nonlocal electromagnetic response). Thus, the all three restrictions on Ginzburg-Landau functional, described in section [1, are excluded.

Let the microscopic magnetic field with a potential $\mathbf{A}(\mathbf{r})$ (with an intensity $\mathbf{H}(\mathbf{r})$ ) exists in the given point of a superconductor:

$$
\mathbf{A}(\mathbf{r})=\sum_{\mathbf{q}} \mathbf{a}_{\mathbf{q}} e^{i \mathbf{q} \mathbf{r}} \Rightarrow \mathbf{H}(\mathbf{r})=\operatorname{rot} \mathbf{A}(\mathbf{r})=i \sum_{\mathbf{q}} \mathbf{q} \times \mathbf{a}_{\mathbf{q}} e^{i \mathbf{q} \mathbf{r}}
$$

Moreover $\mathbf{a}_{-\mathbf{q}}=\mathbf{a}_{\mathbf{q}}^{+}$follows from the reality of $\mathbf{A}$. The energy of magnetic field is

$$
W_{f}=\frac{1}{8 \pi} \int|\mathbf{H}(\mathbf{r})|^{2} d^{3} r=\frac{V}{8 \pi} \sum_{\mathbf{q}}\left(q^{2}\left(\mathbf{a}_{\mathbf{q}} \cdot \mathbf{a}_{\mathbf{q}}^{+}\right)-\left(\mathbf{q} \cdot \mathbf{a}_{\mathbf{q}}\right)\left(\mathbf{q} \cdot \mathbf{a}_{\mathbf{q}}^{+}\right)\right)
$$

The magnetic field affects on a superconductor essentially. In the first place, the distribution of order parameter becomes inhomogeneous. As it was shown in the section III the inhomogeneity leads to the growth of momentum of each element of a pair: $\mathbf{k} \rightarrow \mathbf{k}+\frac{\mathbf{q}}{2},-\mathbf{k} \rightarrow-\mathbf{k}-\frac{\mathbf{q}}{2}$, moreover the order parameter depends on the momentum $\Delta=\Delta(\mathbf{q})$. In the second place, the ordinary momentum must be replaced by the canonical momentum: $\mathbf{k}+\frac{\mathbf{q}}{2} \rightarrow \mathbf{k}+\frac{\mathbf{q}}{2}-\frac{e}{c} \mathbf{a} \mathbf{q}$ and $-\mathbf{k}-\frac{\mathbf{q}}{2} \rightarrow-\mathbf{k}-\frac{\mathbf{q}}{2}+\frac{e}{c} \mathbf{a}_{-\mathbf{q}}$, moreover the order parameter is function of the momentums: $\Delta=\Delta\left(\mathbf{q}, \mathbf{a}_{\mathbf{q}}\right)$. For convenience let's pass to the reference system of center of mass of a pair, where the momentums of particles are equal by modulus, are directed oppositely and are real:

$$
\begin{aligned}
& \mathbf{p}_{01}=\frac{m_{2} \mathbf{p}_{1}-m_{1} \mathbf{p}_{2}}{m_{1} m_{2}}=\mathbf{k}+\frac{\mathbf{q}}{2}-\frac{e}{2 c}\left(\mathbf{a}_{\mathbf{q}}+\mathbf{a}_{\mathbf{q}}^{+}\right) \\
& \mathbf{p}_{01}=-\frac{m_{2} \mathbf{p}_{1}-m_{1} \mathbf{p}_{2}}{m_{1} m_{2}}=-\mathbf{k}-\frac{\mathbf{q}}{2}+\frac{e}{2 c}\left(\mathbf{a}_{\mathbf{q}}+\mathbf{a}_{\mathbf{q}}^{+}\right)
\end{aligned}
$$

because $m_{1}=m_{2}=m$. The hamiltonian of the system has a form:

$$
\begin{aligned}
& \widehat{H}_{\mathrm{eff}}=\sum_{\mathbf{q}} \sum_{\alpha} \sum_{\mathbf{k}} \varepsilon_{q}\left(\mathbf{k}+\frac{\mathbf{q}}{2}-\frac{e}{2 c}\left(\mathbf{a}_{\mathbf{q}}+\mathbf{a}_{\mathbf{q}}^{+}\right)\right) C_{\mathbf{k}+\frac{\mathbf{q}}{2}-\frac{e}{2 c}\left(\mathbf{a}_{\mathbf{q}}+\mathbf{a}_{\mathbf{q}}^{+}\right), \alpha} C_{\mathbf{k}+\frac{\mathbf{q}}{2}-\frac{e}{2 c}\left(\mathbf{a}_{\mathbf{q}}+\mathbf{a}_{\mathbf{q}}^{+}\right), \alpha} \\
& +\frac{1}{2 V} \sum_{\mathbf{q}} \sum_{\alpha, \gamma} \sum_{\mathbf{k}, \mathbf{l}} \lambda(\mathbf{q}) w_{k} w_{l} g_{\alpha \gamma} C_{-\mathbf{l}-\frac{\mathbf{q}}{2}+\frac{e}{2 c}\left(\mathbf{a}_{\mathbf{q}}+\mathbf{a}_{\mathbf{q}}^{+}\right), \gamma} C_{\mathbf{l}+\frac{\mathbf{q}}{2}-\frac{e}{2 c}\left(\mathbf{a}_{\mathbf{q}}+\mathbf{a}_{\mathbf{q}}^{+}\right), \alpha} C_{\mathbf{k}+\frac{\mathbf{q}}{2}-\frac{e}{2 c}\left(\mathbf{a}_{\mathbf{q}}+\mathbf{a}_{\mathbf{q}}^{+}\right), \alpha} C_{-\mathbf{k}-\frac{\mathbf{q}}{2}+\frac{e}{2 c}\left(\mathbf{a}_{\mathbf{q}}+\mathbf{a}_{\mathbf{q}}^{+}\right), \gamma},
\end{aligned}
$$

if $\lambda(\mathbf{q})<0$. The hamiltonian (172) is the generalization of BCS hamiltonian (11) to the cases of the dependence of the interaction constant on coordinates and to the presence of a magnetic field.

The system with the hamiltonian (72) is unstable regarding in Cooper pairing. The mass operator for process of interaction of a fermion with fluctuations of pairing is shown in Fig 4. In analytical representation this diagram has 


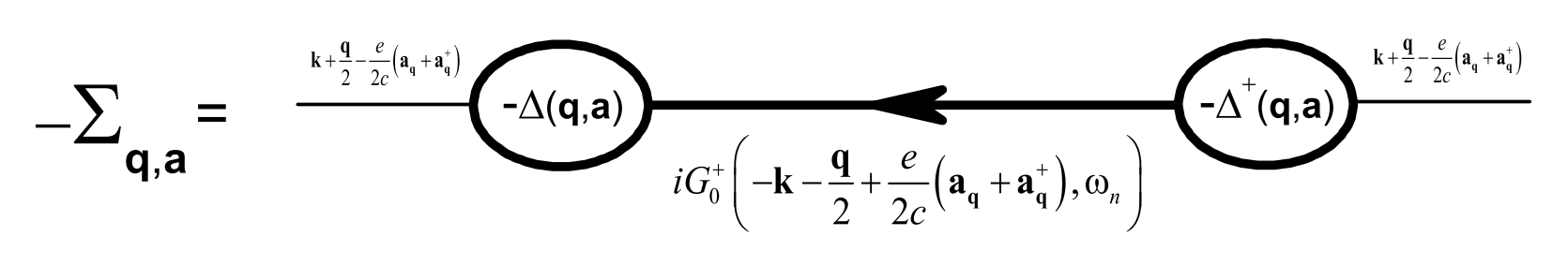

Figure 4: The diagram for the mass operator $\Sigma$ describing the interaction of a charged fermion with fluctuations of pairing in a spatially inhomogeneous system situated in a magnetic field with potential $\mathbf{a}(\mathbf{q})$.

the form:

$$
-\Sigma_{\mathbf{q}, \mathbf{a}}\left(\mathbf{k}, \omega_{n}\right)=-\Delta_{\mathbf{q}, \mathbf{a}} i G_{0}^{+}\left(-\mathbf{k}-\frac{\mathbf{q}}{2}+\frac{e}{2 c}\left(\mathbf{a}_{\mathbf{q}}+\mathbf{a}_{\mathbf{q}}^{+}\right), \omega_{n}\right)\left(-\Delta_{\mathbf{q}, \mathbf{a}}^{+}\right)=\frac{-\Delta_{\mathbf{q}, \mathbf{a}} \Delta_{\mathbf{q}, \mathbf{a}}^{+}}{i \omega_{n}+\varepsilon\left(-\mathbf{k}-\frac{\mathbf{q}}{2}+\frac{e}{2 c}\left(\mathbf{a}_{\mathbf{q}}+\mathbf{a}_{\mathbf{q}}^{+}\right)\right)},
$$

where the free propagator $G_{0}$ is

$$
G_{0}=\frac{1}{i \omega_{n}-\varepsilon\left(\mathbf{k}+\frac{\mathbf{q}}{2}-\frac{e}{2 c}\left(\mathbf{a}_{\mathbf{q}}+\mathbf{a}_{\mathbf{q}}^{+}\right)\right)}=i \frac{i \omega_{n}+\varepsilon\left(\mathbf{k}+\frac{\mathbf{q}}{2}-\frac{e}{2 c}\left(\mathbf{a} \mathbf{q}+\mathbf{a}_{\mathbf{q}}^{+}\right)\right)}{\left(i \omega_{n}\right)^{2}-\varepsilon^{2}\left(\mathbf{k}+\frac{\mathbf{q}}{2}-\frac{e}{2 c}\left(\mathbf{a} \mathbf{q}+\mathbf{a}_{\mathbf{q}}^{+}\right)\right)} .
$$

From Dyson equation we can obtain the dressed propagator:

$$
\frac{1}{G_{0}}=\frac{1}{G_{S}}-i \Sigma_{\mathbf{q}} \Rightarrow G_{S}=i \frac{i \omega_{n}+\varepsilon\left(\mathbf{k}+\frac{\mathbf{q}}{2}-\frac{e}{2 c}\left(\mathbf{a}_{\mathbf{q}}+\mathbf{a}_{\mathbf{q}}^{+}\right)\right)}{\left(i \omega_{n}\right)^{2}-E_{\mathbf{q}, \mathbf{a}}^{2}}
$$

where $E$ is the specter of quasi-particles in an inhomogeneous system situated in magnetic field:

$$
\begin{aligned}
& E_{\mathbf{q}, \mathbf{a}}^{2}=\varepsilon^{2}\left(\mathbf{k}+\frac{\mathbf{q}}{2}-\frac{e}{2 c}\left(\mathbf{a}_{\mathbf{q}}+\mathbf{a}_{\mathbf{q}}^{+}\right)\right)+\left|\Delta_{\mathbf{q}, \mathbf{a}}\right|^{2} \\
& \varepsilon\left(\mathbf{k}+\frac{\mathbf{q}}{2}-\frac{e}{2 c}\left(\mathbf{a}_{\mathbf{q}}+\mathbf{a}_{\mathbf{q}}^{+}\right)\right) \approx \varepsilon(k)+\frac{\mathbf{k} \cdot\left(\frac{\mathbf{q}}{2}-\frac{e}{2 c}\left(\mathbf{a}_{\mathbf{q}}+\mathbf{a}_{\mathbf{q}}^{+}\right)\right)}{m} \equiv \varepsilon \mathbf{q}, \mathbf{a}, \quad|\mathbf{k}| \simeq k_{F},
\end{aligned}
$$

where we have introduced the notations $E_{\mathbf{q}, \mathbf{a}}$ and $\varepsilon_{\mathbf{q}, \mathbf{a}}$ for convenience. Dyson equation can be represented in the form of Gor'kov equations. From these equations the expressions for anomalous propagators follow:

$$
\begin{gathered}
\left(i \omega_{n}-\varepsilon_{\mathbf{q}, \mathbf{a}}\right) G+\Delta_{\mathbf{q}, \mathbf{a}} F^{+}=i \\
\left(i \omega_{n}+\varepsilon_{\mathbf{q}, \mathbf{a}}\right) F^{+}+G \Delta_{\mathbf{q}, \mathbf{a}}=0
\end{gathered} \Rightarrow \begin{gathered}
F_{\mathbf{q}, \mathbf{a}}^{+}=\frac{-i \Delta_{\mathbf{q}, \mathbf{a}}^{+}}{\left(i \omega_{n}\right)^{2}-E_{\mathbf{q}, \mathbf{a}}^{2}} \\
F_{\mathbf{q}, \mathbf{a}}=\left(F_{\mathbf{q}, \mathbf{a}}^{+}\right)^{+}=\frac{i \Delta \mathbf{q}, \mathbf{a}}{\left(i \omega_{n}\right)^{2}-E_{\mathbf{q}, \mathbf{a}}^{2}}
\end{gathered}
$$

If to suppose $\mathbf{q}=0$ and $\mathbf{a}=0$, then we shall have the expressions (18]20).

Free energy of a superconductor is a sum of the following terms:

$$
\Omega=\Omega_{n}+W_{S}-\frac{1}{\beta} S_{S}+\Omega_{\lambda}+W_{\mathbf{f}}(\mathbf{a})
$$

where $W_{S}$ is the kinetic energy of fermions in superconducting phase, $S_{S}$ is the entropy of boholons, $\Omega_{\lambda}=-\frac{1}{\beta} \ln R(\beta)$ is the energy corresponding to an interaction, $W_{f}(\mathbf{a})$ is the energy of magnetic field (70). The expressions for $W_{S}, S$ and $\Omega_{\lambda}$ are obtained from the expression (34) by the replacement $\Delta \rightarrow \Delta_{\mathbf{q}, \mathbf{a}}, E \rightarrow E_{\mathbf{q}, \mathbf{a}}, \varepsilon \rightarrow \varepsilon_{\mathbf{q}, \mathbf{a}}, f \rightarrow f_{\mathbf{q}, \mathbf{a}}$. Hence 
the free energy of a superconductor is

$$
\begin{aligned}
& \Omega=-\frac{2 i}{\beta} \lim _{\tau \rightarrow 0^{-}} \sum_{\mathbf{q}} \sum_{\mathbf{k}} \sum_{n=-\infty}^{n=+\infty} G\left(\mathbf{k}+\frac{\mathbf{q}}{2}-\frac{e}{2 c}\left(\mathbf{a}_{\mathbf{q}}+\mathbf{a}_{\mathbf{q}}^{+}, \omega_{n}\right) e^{-i \omega_{n} \tau} \varepsilon\left(\mathbf{k}+\frac{\mathbf{q}}{2}-\frac{e}{2 c}\left(\mathbf{a}_{\mathbf{q}}+\mathbf{a}_{\mathbf{q}}^{+}\right)\right)\right. \\
& +\sum_{\mathbf{q}} \sum_{\mathbf{k}} \frac{2}{\beta}\left[f_{\mathbf{q}, \mathbf{a}}^{S} \ln f_{\mathbf{q}, \mathbf{a}}^{S}+\left(1-f_{\mathbf{q}, \mathbf{a}}^{S}\right) \ln \left(1-f_{\mathbf{q}, \mathbf{a}}^{S}\right)-f_{\mathbf{q}, \mathbf{a}}^{0} \ln f_{\mathbf{q}, \mathbf{a}}^{0}-\left(1-f_{\mathbf{q}, \mathbf{a}}^{0}\right) \ln \left(1-f_{\mathbf{q}, \mathbf{a}}^{0}\right)\right] \\
& -\sum_{\mathbf{q}} \frac{\lambda(\mathbf{q})}{V} \sum_{\mathbf{k}} w_{k} \frac{1}{\beta} \sum_{n=-\infty}^{n=+\infty} F_{\mathbf{q}, \mathbf{a}}\left(\mathbf{k}, \omega_{n}\right) \sum_{\mathbf{k}} w_{k} \frac{1}{\beta} \sum_{n=-\infty}^{n=+\infty} F_{\mathbf{q}, \mathbf{a}}\left(\mathbf{k}, \omega_{n}\right) \\
& +\frac{V}{8 \pi} \sum_{\mathbf{q}}\left(q^{2}\left(\mathbf{a}_{\mathbf{q}} \cdot \mathbf{a}_{\mathbf{q}}^{+}\right)-\left(\mathbf{q} \cdot \mathbf{a}_{\mathbf{q}}\right)\left(\mathbf{q} \cdot \mathbf{a}_{\mathbf{q}}^{+}\right)\right)
\end{aligned}
$$

where

$$
f_{\mathbf{q}, \mathbf{a}}^{S}(k)=\frac{1}{e^{\beta E} \mathbf{q}, \mathbf{a}^{(k)}+1}, \quad f_{\mathbf{q}, \mathbf{a}}^{0}(k)=\frac{1}{e^{\beta \mid \varepsilon} \mathbf{q}, \mathbf{a}^{(k) \mid}+1}
$$

are occupation numbers of states by boholons, and with help of the term $f_{\mathbf{q}, \mathbf{a}}^{0}$ the normal part of entropy is separated, such that $\Omega_{S}(\Delta=0)=0$.

Unlike Ginzburg-Landau functional the obtained functional of free energy (79) is correct for an arbitrary value of the relation $\Delta(T) / T$, for arbitrary spatial variations of the order parameter $\Delta(\boldsymbol{r})$, for an arbitrary value of a magnetic penetration depth $\Lambda(T)$ in comparison with a coherent length $l_{0}$. The observed value of $\Delta_{\mathbf{q}, \mathbf{a}}$ minimizes the free energy:

$$
\frac{d \Omega}{d \Delta_{\mathbf{q}, \mathbf{a}}}=0 \Longrightarrow(-i) \Delta_{\mathbf{q}, \mathbf{a}}=\frac{\lambda(\mathbf{q})}{V \beta} \sum_{\mathbf{k}} \sum_{n=-\infty}^{n=+\infty} w_{k} F_{\mathbf{q}, \mathbf{a}}\left(\mathbf{k}, \omega_{n}\right)
$$

If we suppose $\mathbf{a}=0$ and neglect by the dependence on $\mathbf{q}$, then we obtain the expression (35). The functional (79) is complicated for an analyze. Let us suppose that $\Delta(\mathbf{r})$ changes in space slowly and let us expand the expression (79) in degrees of $\frac{\mathbf{q}}{2}-\frac{e}{2 c}\left(\mathbf{a}_{\mathbf{q}}+\mathbf{a}_{\mathbf{q}}^{+}\right)$keeping terms which are proportional to the vector in second degree only. In order to simplify our consideration let us suppose that the parameter of interaction $\lambda(\mathbf{q})=$ const. This means that a spatially inhomogeneity of the order parameter is caused by a magnetic field only. Let us consider the high-temperature limit of free energy: $\Delta \beta_{C} \ll 1$ at $T \rightarrow T_{C}$. This means, that the expression (79) can be expended in degrees of $\Delta_{\mathbf{q}, \mathbf{a}}$ :

$$
\begin{aligned}
\Omega=\Omega_{n} & +V \sum_{\mathbf{q}}\left(\alpha(T) \Delta_{\mathbf{q}, \mathbf{a}}^{2}+\frac{1}{2} b \Delta_{\mathbf{q}, \mathbf{a}}^{4}+\gamma\left(\mathbf{q}-\frac{e}{c}\left(\mathbf{a}_{\mathbf{q}}+\mathbf{a}_{\mathbf{q}}^{+}\right)\right)^{2} \Delta_{\mathbf{q}, \mathbf{a}}^{2}\right) \\
& +\frac{V}{8 \pi} \sum_{\mathbf{q}}\left(q^{2}\left(\mathbf{a}_{\mathbf{q}} \cdot \mathbf{a}_{\mathbf{q}}^{+}\right)-\left(\mathbf{q} \cdot \mathbf{a}_{\mathbf{q}}\right)\left(\mathbf{q} \cdot \mathbf{a}_{\mathbf{q}}^{+}\right)\right),
\end{aligned}
$$

where the coefficients $\alpha(T), b$ are determined by the formula (41), and the coefficient $\gamma$ is determined by the formula (56). The expansion (82) has the form of Ginzburg-Landau expansion of free energy in degrees of the order parameter. Observed configurations of the order parameter $\Delta_{\mathbf{q}, \mathbf{a}}$ and magnetic field $\mathbf{a}(\mathbf{q})$ minimizes the free energy:

$$
\begin{aligned}
\frac{\delta \Omega}{\delta \Delta}=0 & \Rightarrow \alpha(T) \Delta_{\mathbf{q}, \mathbf{a}}+b \Delta_{\mathbf{q}, \mathbf{a}}^{3}+\gamma\left(\mathbf{q}-\frac{e}{c}\left(\mathbf{a}_{\mathbf{q}}+\mathbf{a}_{\mathbf{q}}^{+}\right)\right)^{2} \Delta_{\mathbf{q}, \mathbf{a}}=0 \\
\frac{\delta \Omega}{\delta \mathbf{a}}=0 & \Rightarrow \mathbf{j}^{+}(\mathbf{q})=e \gamma \mathbf{q} \Delta_{\mathbf{q}, \mathbf{a}}^{2}-\gamma \frac{e^{2}}{c} \Delta_{\mathbf{q}, \mathbf{a}}^{2}\left(\mathbf{a}_{\mathbf{q}}+\mathbf{a}_{\mathbf{q}}^{+}\right) \\
\frac{\delta \Omega}{\delta \mathbf{a}^{+}}=0 & \Rightarrow \mathbf{j}(\mathbf{q})=e \gamma \mathbf{q} \Delta_{\mathbf{q}, \mathbf{a}}^{2}-\gamma \frac{e^{2}}{c} \Delta_{\mathbf{q}, \mathbf{a}}^{2}\left(\mathbf{a}_{\mathbf{q}}+\mathbf{a}_{\mathbf{q}}^{+}\right)
\end{aligned}
$$

where $\mathbf{j}(\mathbf{q})$ is Fourier component of a current:

$$
\begin{aligned}
\mathbf{J}(\mathbf{r})=\frac{c}{4 \pi} \operatorname{rot} \mathbf{H}(\mathbf{r}) & \Rightarrow \mathbf{j}(\mathbf{q})=-\frac{c}{4 \pi} \mathbf{q} \times \mathbf{q} \times \mathbf{a}_{\mathbf{q}}=-\frac{c}{4 \pi}\left(\mathbf{q}\left(\mathbf{q} \cdot \mathbf{a}_{\mathbf{q}}\right)-\mathbf{a}_{\mathbf{q}} q^{2}\right) \\
& \Rightarrow \mathbf{j}^{+}(\mathbf{q})=-\frac{c}{4 \pi} \mathbf{q} \times \mathbf{q} \times \mathbf{a}_{\mathbf{q}}^{+}=-\frac{c}{4 \pi}\left(\mathbf{q}\left(\mathbf{q} \cdot \mathbf{a}_{\mathbf{q}}^{+}\right)-\mathbf{a}_{\mathbf{q}}^{+} q^{2}\right) .
\end{aligned}
$$


From the equations (84,85) we can see that $\mathbf{j}(\mathbf{q})=\mathbf{j}^{+}(\mathbf{q})$. From the equation (86) we can see that $\mathbf{a}_{\mathbf{q}}=\mathbf{a}_{\mathbf{q}}^{+}$.

If the superconductor is simply connected (without holes, vortexes) we can pass to the transverse gauge of magnetic field: $\mathbf{q} \cdot \mathbf{a} \mathbf{q}=0$. Then the current is $\mathbf{j}(\mathbf{q})=\frac{c}{4 \pi} \mathbf{a}(\mathbf{q}) \mathbf{q}^{2}$. In transverse gauge the functional of free energy has the form:

$$
\Omega=\Omega_{n}+V \sum_{\mathbf{q}}\left(\alpha(T) \Delta_{\mathbf{q}, \mathbf{a}}^{2}+\frac{1}{2} b \Delta_{\mathbf{q}, \mathbf{a}}^{4}+\gamma\left(\mathbf{q}^{2}+\frac{4 e^{2}}{c^{2}} \mathbf{a}_{\mathbf{q}}^{2}\right) \Delta_{\mathbf{q}, \mathbf{a}}^{2}\right)+\frac{V}{8 \pi} \sum_{\mathbf{q}} \mathbf{q}^{2} \mathbf{a}_{\mathbf{q}}^{2}
$$

The equations of extremals are

$$
\begin{aligned}
& \alpha(T) \Delta_{\mathbf{q}, \mathbf{a}}+b \Delta_{\mathbf{q}, \mathbf{a}}^{3}+\gamma\left(\mathbf{q}^{2}+\frac{4 e^{2}}{c^{2}} \mathbf{a}_{\mathbf{q}}^{2}\right) \Delta_{\mathbf{q}, \mathbf{a}}=0 \\
& \mathbf{j}(\mathbf{q})=-8 \gamma \frac{e^{2}}{c} \Delta_{\mathbf{q}, \mathbf{a}}^{2} \mathbf{a}_{\mathbf{q}}
\end{aligned}
$$

The closed currents $\mathbf{q} \cdot \mathbf{j}(\mathbf{q})=0$ screen a magnetic field in a superconductor. The currents is analogy to molecular currents of Ampere. From Eq.(89) one can see, that the value $Q=-8 \gamma \frac{e^{2}}{c} \Delta_{\mathbf{q}, \mathbf{a}}^{2}$ is the kernel of magnetic response. The order parameter is function of $\mathbf{q}$ and $\mathbf{a}(\mathbf{q})$ :

$$
\Delta_{\mathbf{q}, \mathbf{a}}^{2}(T)=\frac{|\alpha(T)|}{b}\left(1-\frac{\gamma}{|\alpha(T)|}\left(\mathbf{q}^{2}+\frac{4 e^{2}}{c^{2}} \mathbf{a}_{\mathbf{q}}^{2}\right)\right) \approx \frac{\Delta^{2}(T)}{\left(1+l^{2}(T)\left(\mathbf{q}^{2}+\frac{4 e^{2}}{c^{2}} \mathbf{a}_{\mathbf{q}}^{2}\right)\right)}
$$

where $q l(T) \ll 1$. From the formula (90) one can see, that the kernel $Q$ is a function of magnetic field. Hence the electrodynamics of a superconductor is nonlinear. The kernel considers both own nonlocality of a superconductor and the nonlocality caused by fluctuations in the critical region (because $l\left(T \rightarrow T_{C}\right) \rightarrow \infty$ ). If to suppose $\Delta=$ const at given temperature, then we shall obtain London equation:

$$
\mathbf{j}(\mathbf{q})=-8 \gamma \frac{e^{2}}{c} \Delta^{2}(T) \mathbf{a} \mathbf{q} \equiv-\frac{c}{4 \pi \Lambda^{2}(T)} \mathbf{a}(\mathbf{q}) \Rightarrow \Lambda^{2}(T)=\frac{c^{2}}{32 \pi e^{2}} \frac{b}{|\alpha(T)| \gamma},
$$

where $\Lambda(T)$ is the magnetic penetration depth in a superconductor.

For research of the nonlocal characteristics of the free energy functional (79) let us consider the low-temperature limit $\Delta \beta \gg 1$ at $T \rightarrow 0$. The value of gap is close to its value at zero temperature $\Delta(T) \leq \Delta_{0}$. Moreover, a magnetic field is weak, such that it changes the gap lightly, that is the magnetic field is much smaller than critical field $H \ll H_{C}$. Either as above, we assume that a change of gap in space is slow. Starting from aforesaid and using the expansion (38) we obtain the free energy:

$$
\Omega=\Omega_{n}+V \sum_{\mathbf{q}}\left(\alpha_{0}(T)+b_{0}(T) \Delta_{\mathbf{q}, \mathbf{a}}+d_{0} \Delta_{\mathbf{q}, \mathbf{a}}^{2}+\gamma\left(\mathbf{q}^{2}+\frac{4 e^{2}}{c^{2}} \mathbf{a}_{\mathbf{q}}^{2}\right) \Delta_{\mathbf{q}, \mathbf{a}}^{2}\right)+\frac{V}{8 \pi} \sum_{\mathbf{q}} q^{2} a_{\mathbf{q}}^{2}
$$

where coefficients $\alpha_{0}(T), b_{0}(T), d_{0}$ are determined by the formulas (39), and coefficient $\gamma$ is determined by the formula (56), the magnetic field is considered in the transverse gauge $\mathbf{q} \cdot \mathbf{a}_{\mathbf{q}}=0$. The observed configurations of order parameter $\Delta_{\mathbf{q}, \mathbf{a}}$ and magnetic field $\mathbf{a}(\mathbf{q})$ minimized the free energy:

$$
\begin{aligned}
& \frac{\delta \Omega}{\delta \Delta}=0 \Rightarrow b_{0}(T)+2 d_{0} \Delta_{\mathbf{q}, \mathbf{a}}+\gamma\left(\mathbf{q}^{2}+\frac{4 e^{2}}{c^{2}} \mathbf{a}_{\mathbf{q}}^{2}\right) \Delta_{\mathbf{q}, \mathbf{a}}=0 \\
& \frac{\delta \Omega}{\delta \mathbf{a}}=0 \Rightarrow \mathbf{j}(\mathbf{q})=-8 \gamma \frac{e^{2}}{c} \Delta_{\mathbf{q}, \mathbf{a}}^{2} \mathbf{a}(\mathbf{q})
\end{aligned}
$$

If in the equation (94) to assume $\Delta=$ const at given temperature, then we shall have London equation again:

$$
\mathbf{j}(\mathbf{q})=-8 \gamma \frac{e^{2}}{c} \Delta^{2}(T) \mathbf{a}(\mathbf{q}) \equiv-\frac{c}{4 \pi \Lambda^{2}(T)} \mathbf{a}(\mathbf{q}) \Rightarrow \Lambda^{2}(T)=\frac{c^{2}}{8 \pi e^{2}} \frac{d_{0}^{2}}{b_{0}^{2}(T) \gamma} .
$$

The equations (9394) allows to generalize London equation. From the equation (93) we can find the value of gap:

$$
\Delta_{\mathbf{q}, \mathbf{a}}(T)=\frac{-b_{0}(T)}{2 d_{0}+2 \gamma\left(\mathbf{q}^{2}+\frac{4 e^{2}}{c^{2}} \mathbf{a}_{q}^{2}\right)}
$$


Then the equation for current has the form:

$$
\mathbf{j}(\mathbf{q})=-8 \gamma \frac{e^{2}}{c} \frac{b_{0}^{2}(T)}{\left(2 d_{0}+2 \gamma\left(\mathbf{q}^{2}+\frac{4 e^{2}}{c^{2}} \mathbf{a}_{\mathbf{q}}^{2}\right)\right)^{2}} \mathbf{a}(\mathbf{q}) \equiv Q(\mathbf{q}, \mathbf{a}) \mathbf{a}(\mathbf{q})
$$

This equation is the nonlocal and nonlinear generalization of London equation in the long wavelength limit $q \rightarrow 0$, because the kernel $Q$ is the function of $\mathbf{q}$ and magnetic field $\mathbf{a}(\mathbf{q})$. However the equation (97) is correct when the magnetic field is much weaker than the critical field $H \ll H_{C}$.

Let us neglect the nonlinearity, that is we suppose that the kernel $Q$ is function of $\mathbf{q}$ only. Then we have:

$$
\mathbf{j}(\mathbf{q})=-8 \gamma \frac{e^{2}}{c} \frac{b_{0}^{2}(T)}{\left(2 d_{0}+2 \gamma \mathbf{q}^{2}\right)^{2}} \mathbf{a}(\mathbf{q})=-8 \gamma \frac{e^{2}}{c} \frac{\Delta_{0}^{2}(T)}{\left(1+\frac{\gamma}{d_{0}} \mathbf{q}^{2}\right)^{2}} \mathbf{a}(\mathbf{q}) \approx-8 \gamma \frac{e^{2}}{c} \frac{\Delta_{0}^{2}(T)}{\left(1+l_{0}^{2} \mathbf{q}^{2}\right)} \mathbf{a}(\mathbf{q}) \equiv Q(q) \mathbf{a}(\mathbf{q}),
$$

where $l_{0} q \ll 1, \frac{\gamma}{d_{0}}=\frac{l_{0}^{2} \nu_{F}}{\nu_{F}}=l_{0}^{2}$ is the coherent length at temperature $T=0$. Thus we have the nonlocal kernel $Q(q)$, where radius of nonlocality is equal to the coherent length $l_{0}$. This result corresponds to nonlocal Pippard electrodynamics (in the long wave limit). This fact proves a nonlocality of the obtained free energy functional (79). For generalization in the case of large value $l_{0} q$ it is necessary to expand the free energy (79) in degrees of $\mathbf{q}$.

\section{CONCLUSION.}

In this paper we developed the microscopic approach for a finding of the free energy functional of a superconductor with help of direct calculation of a vacuum amplitude. The functional is calculated on the dressed propagators, which takes into account the interaction of a free fermion with the fluctuations of pairing (with the condensate of pairs). As a result of such interaction the dispersion law of quasi-particles is changed and anomalous propagators appear. This means, that a spontaneous symmetry breakdown takes place. After consideration of the interaction of particles with the fluctuations of pairing all characteristics of a system must be calculated over the new vacuum with the broken symmetry. As a result, the free energy is function of the amplitudes $\Omega=\Omega\left(\Delta \Delta^{+}\right)$, and their observed values minimize the free energy.

With help of the developed microscopic approach we have obtained the free energy of a spatially inhomogeneous superconductor (the system with a spatially inhomogeneous distribution of the order parameter $\Delta(\mathbf{r})$ ) for all temperatures. The cause of the inhomogeneity is the dependence of the interaction constant of electrons on coordinates and the influence of a magnetic field. The obtained expression for the free energy is valid for arbitrary spatial variations of the order parameter. In the long-wave limit the functional is similar to Ginzburg-Landau expansion, but the coefficients of expansion depend on coordinates. Moreover, the obtained expression shows the nonlocality of the order parameter: the value of energy gap in a point is determined by the distribution of the energy gap in some neighborhood of this point.

Above-mentioned results have been generalized on the case of presence of a magnetic field and a current. The analysis of the obtained free energy functional (79) shows, that the nonlocality of a magnetic response of a superconductor is the result of the nonlocality of order parameter. The equations of superconductor's state are extremals of the functional, and they are obtained by variation over the gap $\Delta$ and the magnetic field $\mathbf{a}$. In the high-temperature limit the obtained equations are analogous to Ginzburg-Landau equations. In the low-temperature limit the equations show the nonlocal connection between the magnetic field and the current according to the Pippard low.

[1] G. A. Gogadze, A. N. Omel'yanchuk, Fizica nizkikh temperatur 22, No.6, p. 648-651 (1996)

[2] Gogadze G. A., Fizica Nizkikh Temperatur 21, No.2, p. 177-182 (1995)

[3] Kapaev A. V., Kopaev Yu. V., JETP Letters 68, No.3, p. 211-216 (1998)

[4] I.N. Askerzade, Physics-Uspekhi 52, p. 977-988 (2009)

[5] W.E. Lawrence, S. Doniach, in Proc. of the 12th Intern. Conf. on Low Temperature Physics, Kyoto, 1970 (Ed. E Kanda) (Tokyo: Kcegaku, 1971) p. 361

[6] Ovchinnikov Y. N., Kresin V. Z. Eur. Phys. J. B 45, No.1 p.5-7 (2005)

[7] Ovchinnikov Y. N., Kresin V. Z. Eur. Phys. J. B 47, No.3 p.333-336 (2005)

[8] Kresin V. Z., Ovchinnikov Y. N. Phys. Rev. B 81, No.21 p.214505 (2010)

[9] I.N. Khlyustikov, A.I. Buzdin, Physics-Uspekhi 31, p. 409433 (1988)

[10] Yu.M. Ivanchenko, T.K. Soboleva, JETP letters. 51, No.2, p.114-117 (1990). 
[11] Yu.M. Ivanchenko, T.K. Soboleva. Phys. Lett. A147, No.1, p.65-69 (1990).

[12] R.G. Mints, I.B. Snapiro. Phys. Rev. B51, No.5, p.3054-3057 (1995).

[13] A. Gurevich. Phys. Rev. B46, No.5, p.3187-3190 (1992).

[14] V.P. Silin., JETP letters. 58, No.9, p.701-704 (1993).

[15] V.P. Silin., JETP letters. 60, No.6, p.460-463 (1994).

[16] P.G. Gennes, Superconductivity of metall and alloys (W. A. Benjamin, inc., New York - Amsterdam, 1968).

[17] L. Teword, Phys. Rev. E 132, No2, p.595 (1963).

[18] N.R. Werthamer, Phys. Rev. E 132, No2, p.663 (1963).

[19] J.R. Hook, J.R. Waldram, Proc. R. Soc. Lond. A. 334 No.1597, p.171-192 (1973)

[20] Richard D. Mattuk, A guide to feynman diagrams in the many-body problem (H. C. Oersted Institute University of Copenhagen, Denmark, 1967).

[21] R. D. Mattuk, B.Johansson, Advances in Physics 17, p.509 (1968).

[22] M.V. Sadovskii, Superconductivity and Localization (World Scientific, Singapore, 2000).

[23] A. B. Migdal, Theory of Finite Fermi Systems and Applications to Atomic Nuclei (Interscience Publishers, New York, 1967)

[24] D. Pines, The many-body problem (University of Illinois, New York, 1961) 